Historic, Archive Document

Do not assume content reflects current scientific knowledge, policies, or practices. 



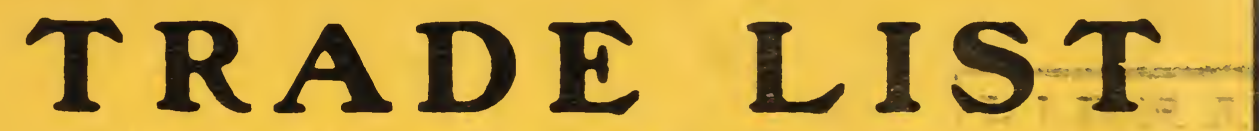

Russellville Nurseries

Fall 1931 - Spring 1932

For the Use and Information of

Nurserymen and Dealers

at

WHOLESALE

File for Future Reference

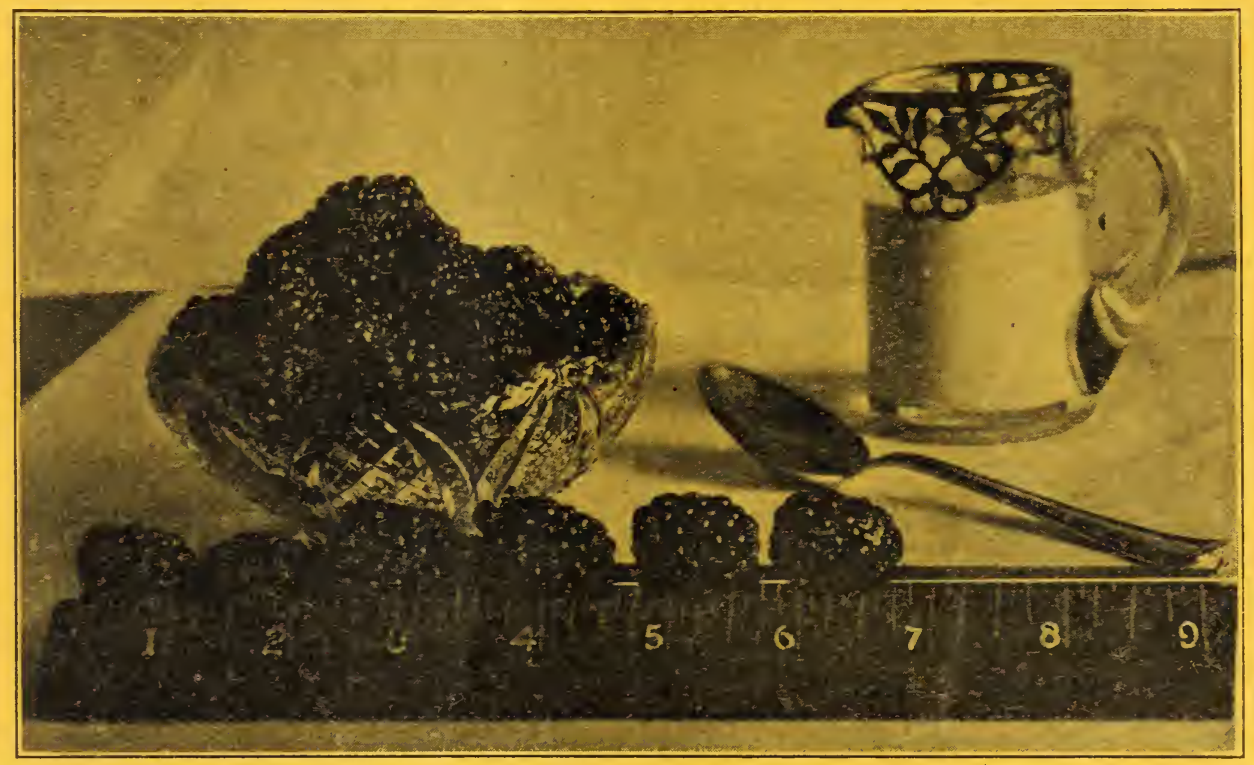

One of the Latest Contributions to the "Small Fruit" Market. A Money-Maker for You and Your Trade. For Information, See Page Seven.

\section{RUSSELLVILLE NURSERIES}

TAbor 0204

East 96th and Burnside Streets

Portland, Oregon

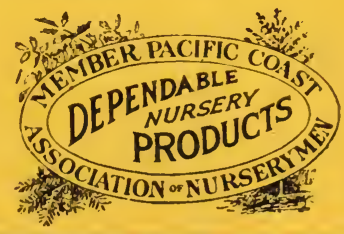




\section{AN INVITATION}

Nurserymen of Oregon Are Entertaining the PACIFIC COAST ASSOCIATION OF NURSERYMEN, at Portland, in September, 1932.

We Sincerely Trust You Will Plan Now to Attend This Convention.

It Will Be Time and Money Well Expended. We Are Counting on You.

Russellville Nurseries

\section{2 - GEORGE WASHINGTON - 1932}

\section{GEORGE WASHINGTON BI-CENTENNIAL ANNIVERSARY}

$$
\text { "The Year } 1932 \text { Is Up to You" }
$$

Cash In on This $\$ 10,000,000$ Tree Planting Program

Get in touch with your local Garden Clubs, Schools, Churches, Patriotic Groups, Civic Clubs and your neighbors. Have them plant trees in commemoration of the birth of George Washington. Plant the trees now, and then arrange to have them dedicated some time during the year 1932.

\section{GET YOUR SHARE OF THIS WORK}

Let Us Help You 


\section{RUSSELLVILLE NURSERIES}

Portland, Oreson

Dear Sirs:

I desire to have my name continued on your mailing list. Am especially interested in some of the following:-

My specialty line is

I have the following stock for sale

$$
\text { Very truly, }
$$




\section{BUSINESS REPLY POST CARD}

NO POSTAGE STAMP NECESSARY IF MAILED IN THE UNITED STATES

2c.- WILL BE PAID BY 2c.

RUSSELLVILLE NURSERIES

EAST BURNSIDE ST. AT 96TH

P. O. BOX 5107

PORTLAND,OREGON

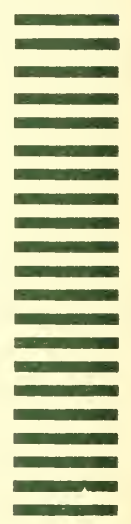




\section{Greetings}

We are here for "Service" and if we can be of assistance to you in any way, please feel at liberty to call.

Nothing would please us more than to have you give our nurseries a personal visit. Come and see us first hand. It will do us both good to get better acquainted.

Send us your Surplus List and your Catalog. Oftentimes we are able to use other stock, for we sell at retail as well as wholesale.

Keep this Price Book for reference so you can get at it handily.

Drop us a line. Our address and phone number is:

\section{RUSSELLVILLE NURSERIES \\ TAbor 0204}

E. Burnside at 96th St. Portland, Oregon 


\section{TERMS}

Prices herein are net F. O. B. Portland, Oregon, when packed in bulk, or if boxed or baled, cost of such packing will be added when invoiced.

Cash or satisfactory reference before shipment is required. To established Nurserymen who are known to us or who give satisfactory references, a credit of 60 days from date of invoice will be given, a discount of $3 \%$ for cash with order, $2 \%$ in 10 days, $1 \%$ in 30 days will be allowed. All bills are due and payable sixty days from date of invoice. Interest at the rate of $8 \%$ per annum will be charged on all past due accounts.

Prices quoted are for first-class stock in the grades named, 5 of a variety will be furnished at 10 rate, 50 at 100 rate, and 500 at 1000 rate.

Grade standards adopted by the P. C. A. of N. are followed by us. Orders should conform to same.

If it is desired that we send another variety when out of the kind ordered, instruct us accordingly, as we do not substitute unless so ordered.

Digging and shipping season begins with us in October, and continues into April with but little interruption on account of climatic conditions.

\section{GUARANTEE OF GENUINENESS}

We exercise care to have our trees and plants true to name and will upon proper proof .replace free any trees or plants that prove untrue to label. It is, however, understood and agreed between the purchaser and ourselves that we are not to be held liable for any sum greater than that paid us for such trees that may prove untrue.

Orders are accepted by us only upon the mutual agreement between the purchaser and ourselves that we shall not be held responsible for other damages.

Claims, arising from any cause, must be made promptly on receipt of shipment. We cannot entertain claims made after stock has been accepted. All claims must be filed within ten days after shipment has been received from carrier.

\section{NOTICE}

Ownership passes and all Nursery stock shipped by us is at the risk of purchaser upon being delivered in good condition to carrier at point of shipment. Any and all claims for damage in transit should be filed immediately with agent of carrier at point of delivery.

Certificate of Horticultural Inspection will accompany each shipment.

This trade list cancels all previous prices and quotations made.

All orders are accepted by us on condition that they shall be void should injury befall the stock from any cause over which we have no control.

State plainly in ordering whether we shall ship by Freight, Express, or Parcel Post. If by Freight, name transportation line over which shipment is to go.

Supply and demand ever will be the yardstick that measures values. The supply of tomorrow may change the value of yesterday in any commodity and also the demand of tomorrow may quickly change the price value of yesterday. We are always ready to meet the conditions of today. We reserve the right to change our prices without notice.

Address: Russellville Nurseries,

East 96 th and Burnside Streets, Portland, Oregon.

Season of 1931-32. 


\section{Wholesale Price List}

\section{Russellville Nurseries \\ Season of 1931.1932 \\ Fruit Trees}

Quotations herein are for one and two year fruit trees. We can supply older and larger stock and will be pleased to quote special on other grades wanted.

\section{Apples and Crabs}

$\begin{array}{lcccc} & \text { Per } & \text { Per } & \text { Per } \\ \text { Caliper } & \text { Height } & 10 & 100 & 1000\end{array}$

2 year $11 / 16$ in. and up, $5 \mathrm{ft}$. and up $\$ 2.75 \$ 25.00 \$ 225.00$ 2 year $9 / 16$ to $11 / 16$ in., 4 ft. and up $2.50 \quad 22.50 \quad 200.00$

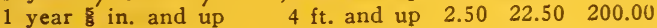

1 year $\frac{1}{2}$ to $\frac{5}{8} \mathrm{in} . \quad 4 \mathrm{ft}$. and up $2.25 \quad 20.00 \quad 175.00$

$\begin{array}{lllll}1 \text { year } \frac{3}{6} \text { to } \frac{1}{2} \mathrm{in} . & 3 \mathrm{ft} \text {. and up } & 2.00 & 17.50 & 150.00\end{array}$

$\begin{array}{ll}\text { Arkansas Black } & \text { Red Delicious } \\ \text { Baldwin } & \text { Red Gravenstein } \\ \text { Delicious } & \text { Red June } \\ \text { Early Harvest } & \text { Red Rome Beauty } \\ \text { Fameuse } & \text { Red Siberian } \\ \text { Gravenstein } & \text { Rome Beauty } \\ \text { Grimes Golden } & \text { Spitzenburg } \\ \text { Hyslop } & \text { Stayman Winesap } \\ \text { Jonathan } & \text { Talman Sweet } \\ \text { King } & \text { Transcendant } \\ \text { King David } & \text { W. W. Pearmain } \\ \text { King Tompkins Co. } & \text { Wagener } \\ \text { Mammoth Blacktwig } & \text { Wealthy } \\ \text { McIntosh Red } & \text { White Astrachan } \\ \text { Northern Spy } & \text { Winesap } \\ \text { Oldenburg } & \text { Winter Banana } \\ \text { Orenco } & \text { Yellow Bellflower } \\ \text { Ortley } & \text { Yellow Newton } \\ \text { R. I. Greening } & \text { Yellow Transparent } \\ \text { Red Astrachan } & \text { Yellow Siberian } \\ & \end{array}$

Red Astrachan

\section{Pears}

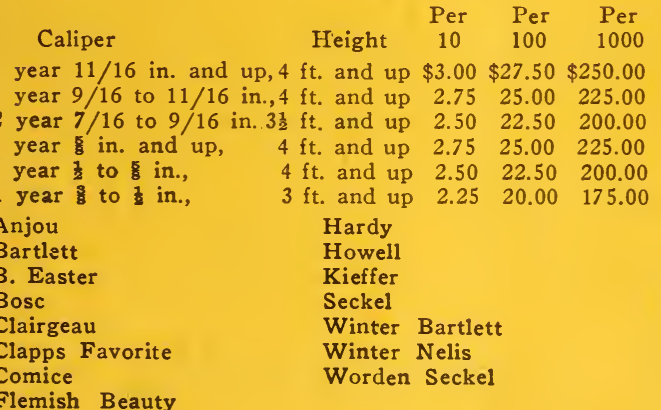

\section{Cherries}

Caliper

16 in. and up $5 \mathrm{ft}$. and up $\$ 5.00 \$ 45.00 \$ 400.00$

2 yr. $9 / 16$ to $11 / 16$ in. $4 \mathrm{ft}$. and up $4.00 \quad 35.00 \quad 300.00$

1 yr. $5 / 8$ in. and up $4 \mathrm{ft}$. and up $4.00 \quad 35.00 \quad 300.00$

1 yr. $1 / 2$ to $5 / 8$ in. $4 \mathrm{ft}$. and up $3.50 \quad 30.00 \quad 250.00$

1 yr. $3 / 8$ to $1 / 2$ in. $3 \mathrm{ft}$. and up $3.00 \quad 30.00 \quad 200.00$

1 yr. $1 / 4$ to $3 / 8$ in.

\section{Bing}

Black Republican

Black Ta:tarian

Early Richmond

English Morello

Lambert $\begin{array}{llll}3 \mathrm{ft} . \text { and up } & 3.00 & 30.00 & 200.00 \\ 2 \mathrm{ft} \text {. and up } & 2.50 & 20.00 & 150.00\end{array}$

Late Duke
May Duke
Montmorency, Lg.
Oregon
Royal Ann
Waterhouse

\section{Peaches}

C'aliper

1 yr. $\delta$ in. and up, $4 \mathrm{ft}$. and up.

$\$ 2.75 \$ 25.00 \$ 225.00$

ft. and up......... $2.25 \quad 20.00 \quad 175.00$

1 yr. $\frac{3}{8}$ to $\frac{1}{2}$ in. $3 \mathrm{ft}$. and up........ $2.00 \quad 17.50 \quad 150.00$

$1 \mathrm{yr} . \frac{1}{4}$ to $\frac{3}{8} \mathrm{in.} \quad 2 \mathrm{ft}$. and up........ $1.75 \quad 15.00 \quad 125.00$

Admiral Dewey_ Johnson Cling

Alexander

Carmen

Champion

Crawfords Early

Crawfords Late

Crosby

Early Charlotte

Early Elberta

Elberta

Fitzgerald

Foster

George IV

Gillingham

Globe

Hales Early

Heath Cling

J. H. Hale

Indian Blood

\section{Plums and Prunes on Myrobolan and Peach}

\section{Caliper}

$\begin{array}{cccc} & \text { Per } & \text { Per } & \text { Per } \\ \text { Height } & 10 & 100 & 1000\end{array}$

2 yr. $11 / 16$ in. and up, $5 \mathrm{ft}$. and up $\$ 2.75 \$ 25.00 \$ 225.00$ 2 yr. $9 / 16$ to $11 / 16$ in., $5 \mathrm{ft}$. and up $2.50 \quad 22.50 \quad 200.00$ 1 yr. $5 / 8$ in. and up, $6 \mathrm{ft}$. and up $2.50 \quad 22.50 \quad 200.00$ 1 yr. $1 / 2$ to $5 / 8$ in., $4 \mathrm{ft}$. and up $2.25 \quad 20.00 \quad 175.00$

\section{Plums on Peach}

Beauty
Burbank
Climax
Columbia
Diamond
Formosa
Gaviota
Grand Duke
Green Gage

Hungarian Prune
Maynard
Peach
Red June
Reine Claude
Santa Rosa
Satsuma
Wickson

Blue Damson

Plums on Myrobolan

Bradshaw

Burbank

Climax

Damson

Green Gage

Peach

Reine Claude
Santa Rosa
Satsuma
Shrop. Damson
Washington
Wickson
Yellow Egg

Reine Claude Satsuma

Shrop. Damson

Wickson

Yellow Egg

\section{PRINLEW PLUM}

New and Different See Description, Page 7 


\section{Apricots on Peach and Myrobolan}

Caliper

1 yr. $\frac{5}{8}$ in. and up,

$1 \mathrm{yr} . \frac{1}{2}$ to $\frac{5}{8}$ in.

$1 \mathrm{yr} . \frac{3}{8}$ to $\frac{1}{2}$ in.

1 yr. $\frac{1}{4}$ to $\frac{3}{8}$ in.

Blenheim

Lewis

Moorpark

1 year $\frac{1}{2}$ in. and up,

1 year $\frac{3}{8}$ to $\frac{1}{2}$ in.

Boston

New White

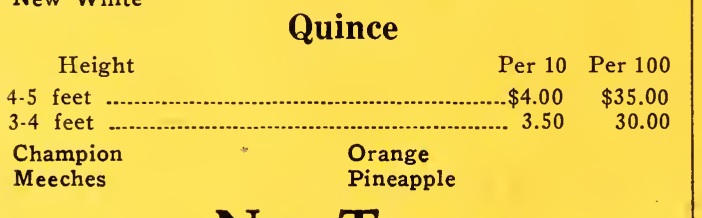

\section{Nut Trees}

\section{Almond on Peach}

Caliper

5 in. and up

$\frac{1}{2}$ to $\frac{5}{8}$ in.,

$\frac{3}{8}$ to $\frac{1}{2}$ in.,

$\frac{1}{4}$ to $\frac{3}{8}$ in.,

Drakes Seedling

I. X. L.

Height

6 to $8 \mathrm{ft}$.

5 to $6 \mathrm{ft}$.

Height

8 to 10 feet

6 to 8 feet

5 to 6 feet

4 to 5 feet

3 to 4 feet

American Sweet

Italian

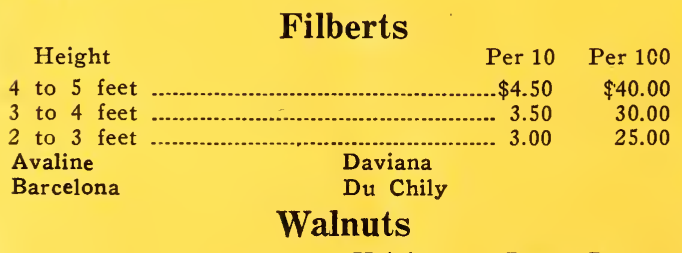

Height Per 10 Per 100

Franquette Grafts, 8 to $10 \mathrm{ft}$. on Cal. Blk. $\$ 12.50 \$ 100.00$

Franquette Grafts, 6 to $8 \mathrm{ft}$. on Cal. Blk. $10.00 \quad 80.00$

Franquette Grafts, 4 to $6 \mathrm{ft}$. on Cal. Blk. $8.50 \quad 60.00$

Franquette Grafts, 3 to $4 \mathrm{ft}$. on Cal. Blk. $6.00 \quad 40.00$

\section{Small Fruits}

\section{Grapes}

\section{American Varieties}

Per Per Per

Campbell's Early ....2 year No. $1 \ldots \ldots . . . \$ 1.50 \$ 12.50 \$ 100.00$ Concord …...............2 year No. 1........ $1.00 \quad 8.00 \quad 70.00$

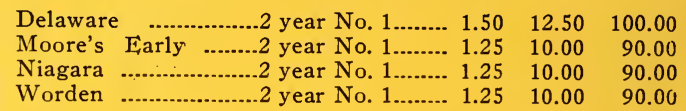

\section{European Varieties}

All 2 year No. 1 $\$ 1.25 \$ 10.00 \$ 80.00$ Black Hamburg Malaga

Thompson's Seedless

Muscat

Tokay

\section{Raspberries}

$\begin{array}{ccc}\text { Per } & \text { Per } & \text { Per } \\ 10 & 100 & 1000\end{array}$

Cumberland, black, transplants...........\$.50 $\$ 4.00 \$ 35.00$

Gregg, black, transplants...................... $\quad .50 \quad 4.00 \quad 35.00$

Plum Farmer, black, transplants.......

Columbian, purple, transplants........... $\quad .60 \quad 4.00 \quad 35.00$

$\begin{array}{lllll}\text { Cuthbert, red, suckers................................... } & .25 & 1.00 & 7.00\end{array}$

$\begin{array}{llll}\text { Marlboro, red, suckers......................................... } & .30 & 1.25 & 10.00\end{array}$

St. Regis, red, suckers.............................. $.50 \quad 3.50 \quad 25.00$

\section{Blackberries}

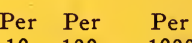

$10100 \quad 1000$

Cory's Thornless, transplants............. \$1.50 $\$ 12.00 \$ 100.00$

Evergreen, suckers and tips................ $\quad .50 \quad 3.00 \quad 25.00$

Evergreen, transplants ….................... $\quad .60 \quad 5.00 \quad 40.00$

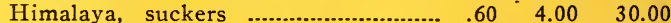

Himalaya, transplants .............................. $\quad .70 \quad 6.00 \quad 45.00$

Lawton, suckers …............................... $\quad .50 \quad 3.00 \quad 25.00$

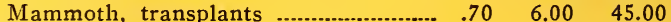

Rathbun, suckers …............................ $.50 \quad 3.00 \quad 25.00$

Lucretia Dewberry, tips ............................... $.50 \quad 3.00 \quad 25.00$

Lucretia Dewberry, transplants........... $\quad .60 \quad 4.00 \quad 30.00$

\section{Loganberries}

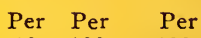

$\begin{array}{lll}10 & 100 & 1000\end{array}$

Transplants

$\$ .70 \$ 6.00 \$ 50.00$

Tips, for spring delivery..................... $3.50 \quad 25.00$

\section{Strawberries}

Clarks Seedling Marshail

Ettersburg New Oregon

Gold Dollar Premier

Magoon Senator Dunlop

\section{Everbearing Varieties}

Mastodon ...................................................\$1.50 \$12.00

Progressive

\section{Asparagus}

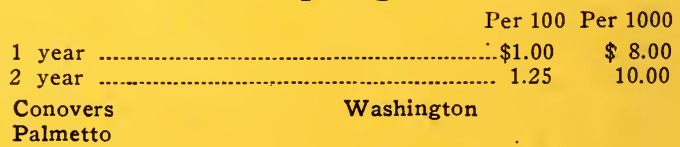

\section{Rhubarb \\ Crown Division}

Maminoth Victoria

Fer 10 Per 100 Per 1000 Early Strawberry
$\$ .80 \$ 5.00 \$ 35.00$

$1.00 \quad 7.00 \quad 50.00$ 


\section{The Young Berry}

The Young Berry is a money-maker-it has been planted extensively in California and has proven itself commercially.

The Young Berry is quite dark in color, apparently free from heavy core and seeds, and the flavor is delicious.

The habit of growth is similar to that of the Logan-berry. Their care and cultivation does not differ much from that of the Logan-berry.

Here is a splendid chance to "cash in" on this new berry plant. Your customers are expecting something that will yield them quick returns. The Young. Berry will serve the purpose, for it will can, dry or sell as fresh fruit. It is our opinion that for extract and juice purposes, it cannot be surpassed.

PUSH THIS PLANT NOW-The fruit market is ready for something new. Take advantage of it and get busy.

We can supply the Young Berry plants at reasonable cost. Many orders have already been booked for Early Spring Delivery.

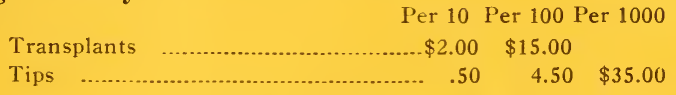

(See cul on front cover)

ereeres

\section{The Prinlew Plum}

The Prinlew Plum was originated by H. A. Lewis, the founder and owner of Russellville Nurseries.

The original tree still stands, having come from seed whicih was evidently double pollinated from the parent tree. This insures a standard fruit with absolutely no possibility of reversion.

One of the most delicious fruits known, with a peach flavor, juicy and sweet.

Can be eaten before it is fully ripe, which makes it very desirable over a longer season.

Fruit ripens in August, when there is a general scarcity of tree-fruits.

Splendid to eat from hand or for canning. Eight of these plums in the $2 \mathrm{I} / 2^{-}$pound ordinary can. The fruit is larger than the well known Bradshaw Plum and far superior in flavor. When canned, they" have a delightful sweet twangy flavor that cannot be equalled by any other prune or plum.

One of the best road-side market plums known. The splendid light blue color with the light maroon-red cheek makes it very attractive and they sell on sight. Your customers come back for more.

The tree is a regular and heavy bearer-thrifty and sturdy grower-and has been known to do well under many different climatic conditions.

It is a money-maker for the owner. You cannot afford to be without it.

Introductory price on extra first grade tree. Dealers may obtain these trees at the following special prices. Less than 10 quantity, $\$ 1.00$ each. We suggest that you order more than ten, for in this way we can allow you the hundred rate of 35 cents each.

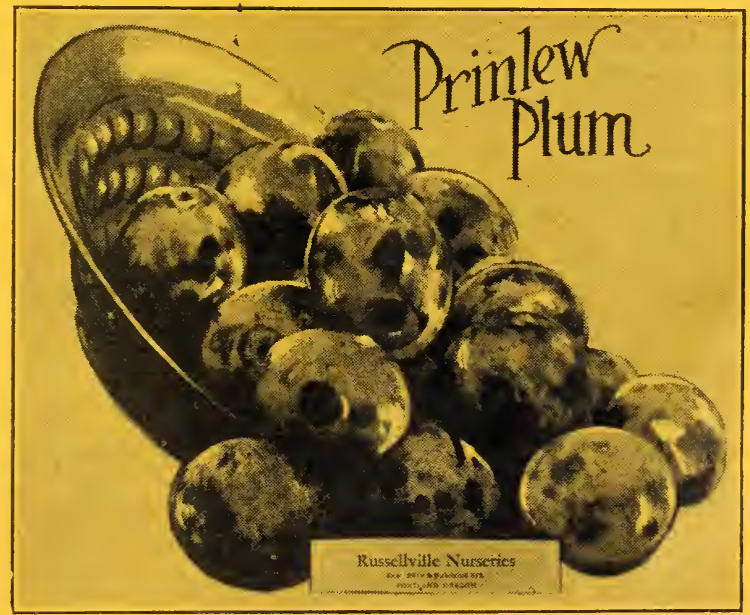

You can easily afford to boost the "Prinlew Plum." One tasle of this luscious fruit will convince you of this fact. Let us tell you more about it! 


\section{Seedlings}

Oregon-grown under most ideal soil and climatic conditions by white American labor. Superior to imported stocks. Dug, graded, packed and shipped direct to you by the grower. Start the foundation of your nursery right, then you'll be right all along the way.

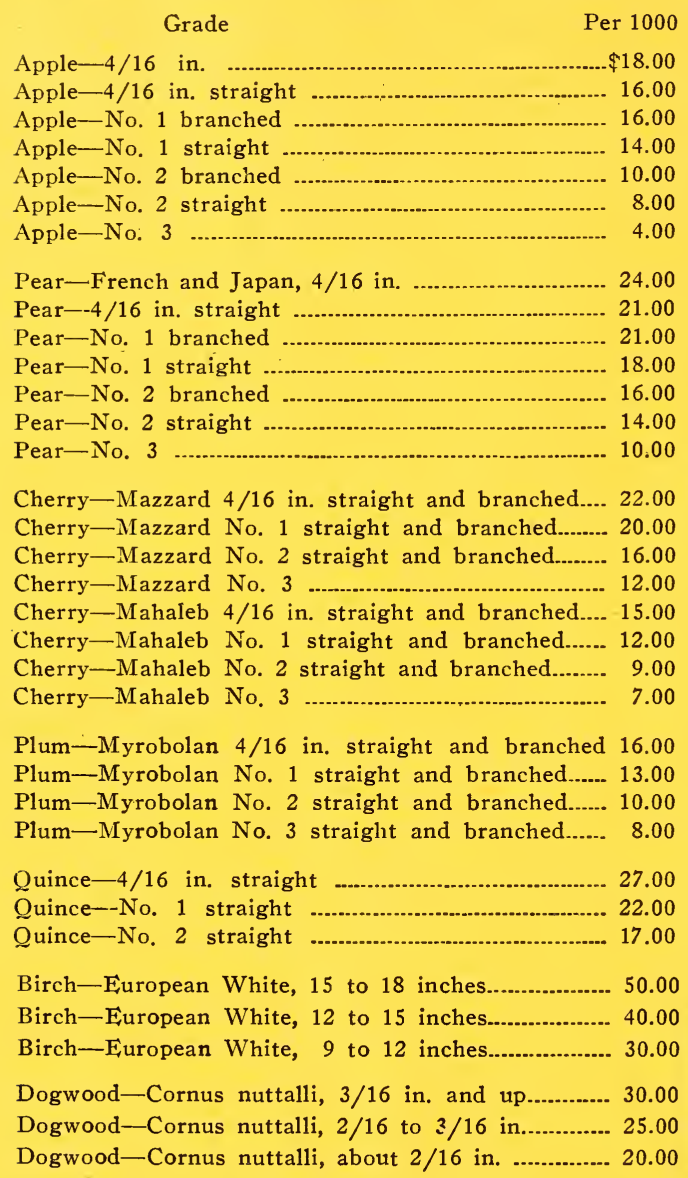

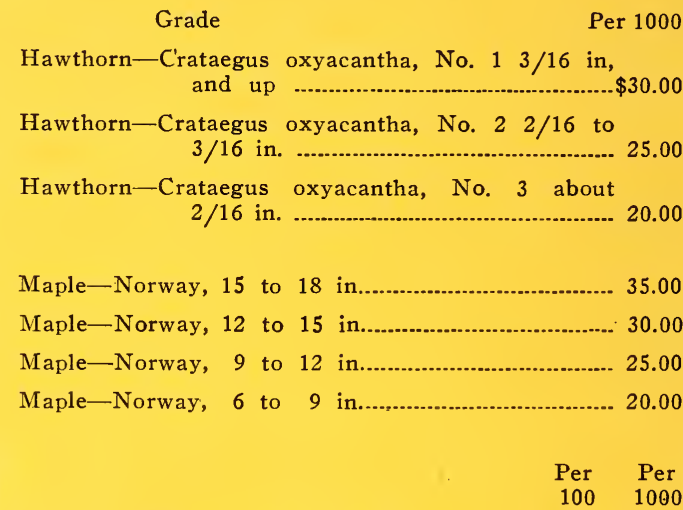

Elm-Chinese-Ulmus Pumila, 3 to $4 \mathrm{ft} \ldots . . . \$ 7.50 \$ 50.00$ Elm-Chinese-Ulmus Pumila, 2 to $3 \mathrm{ft}$.... $5.00 \quad 35.00$ Elm-Chinese-Ulmus Pumila, 18 to 24 in..... 3.5025 .00 Elm-Chinese-Ulmus Pumila, 12 to 18 in..... $3.00 \quad 20.00$ Elm-Chinese-Ulmus Pumila, 9 to 12 in..... 2.0012 .00 Rooted Manetti, Odorata and Rugosa, 5 to 9 M. M. 20.00

\section{NORWAY MAPLE SEED}

50 to $100 \mathrm{lbs}$. $40 \mathrm{c}$ per $1 \mathrm{~b}$. 100 to $250 \mathrm{lbs}$. $35 \mathrm{c}$ per $1 \mathrm{~b}$. 250 to $500 \mathrm{lbs}$. $30 \mathrm{c}$ per $1 \mathrm{~b}$.

\section{SYCAMORE MAPLE SEED}

10 to $50 \mathrm{lbs}$. $85 \mathrm{c}$ per $1 \mathrm{~b}$. 50 to $100 \mathrm{lbs}$. $75 \mathrm{c}$ per $1 \mathrm{~b}$. 100 to $250 \mathrm{lbs}$. $60 \mathrm{c}$ per $\mathrm{lb}$.

250 lbs. and up $50 \mathrm{c}$ per $1 \mathrm{~b}$.

\section{Ornamental and Shade Trees}

\section{ASH-}

Black, (Fraxinus nigra)

Green, (Fraxinus lanceolata)

White, (Fraxinus americana)

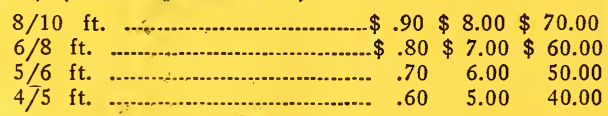

Mountain European, (Sorbus a ucuparia)

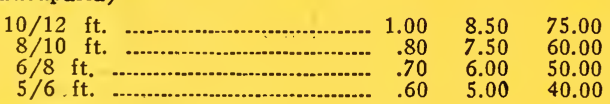

\section{BIRCH-}

Cutleaf Weeping, (Betula laciniata)

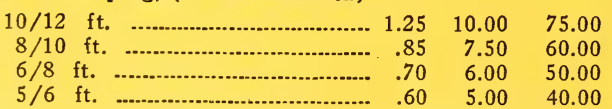

European White, (Betula alba) Each Per 10 Per 100 $\begin{array}{rl}10 / 12 & \mathrm{ft} \text {. } \\ 8 / 10 \mathrm{ft} & .\end{array}$ $6 / 8 \mathrm{ft}$. $5 / 6 \mathrm{ft}$.

$\begin{array}{lll}.85 & 7.00 & 55.00 \\ .75 & 6.00 & 45.00 \\ .60 & 5.00 & 35.00 \\ .50 & 4.00 & 30.00 \\ .40 & 3.00 & 20.00\end{array}$

\section{BOXELDER-}

Boxelder, (Acer negundo) $8 / 10 \mathrm{ft}$. $6 / 8 \mathrm{ft}$.

Golden Variegated (A. Auratum) $4 / 5 \mathrm{ft}$. $\begin{array}{lll}.60 & 5.00 \quad 40.00\end{array}$

Silver Variegated, (A. negundo variegata)

$5 / 6 \mathrm{ft}$.

$4 / 5 \mathrm{ft}$.

$\begin{array}{lll}.70 & 6.00 & 50.00\end{array}$

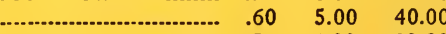

FAGUS BEACH PURPLE

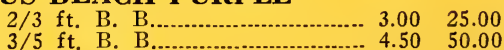

$5 / 6 \mathrm{ft}$. B. B........................... $6.50 \quad 55.00$ 


\section{CATALPA-}

Western, (Catalpa speciosa) Each Per 10 Per 100 $8 / 10$ ft. .................................. $1.00 \quad 8.50 \quad 75.00$

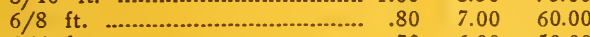

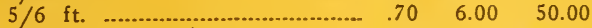
$4 / 5$ ft. ............................................ $60 \quad 5.00 \quad 40.00$

Bungei

6 ft. stems …........................... $1.10 \quad 9.00 \quad 75.00$

5 ft. stems …........................ $\quad .90 \quad 7.50 \quad 60.00$

\section{CHERRY-}

Double Rose, Amanagawa Zakura

Dble. Pink Hosokawa

Dble. Red, J. H. Viettch

Dble. Pink, Kwazan

Dble. White, Mt. Fuji

Dble. Pink Naden

Dble. Blush, Ruth Wohlert

$6 / 8$ ft. 2 yr.

$\begin{array}{rrr}1.50 & 12.50 & 100.00\end{array}$

$5 / 6$ ft. 2 yr.

$\begin{array}{rrr}1.25 & 10.00 & 85.00\end{array}$

$4 / 5$ ft. 2 yr.

$1.00 \quad 8.50$

$5 / 6$ ft. 1 yr.

1.00

$4 / 5$ ft. 1 yr.

75.00

$3 / 4$ ft. 1 yr.

$.90 \quad 7.50$

ble. Rose, Kofugen

$3 / 4 \mathrm{ft}$.

$2 / 3$ ft.

$\begin{array}{lr}1.00 & 10.00\end{array}$

$.85 \quad 7.50$

Weeping, Prunus subhirtella pen-

dula Flg.

$6 / 7$ ft. stem, No. 1 heads........ $1.75 \quad 15.00 \quad 125.00$

$5 / 6 \mathrm{ft}$. stem, No. 1 heads......... $1.50 \quad 12.50 \quad 100.00$

\section{CHESTNUT-}

Evergreen), Chinquapin,

(Castanea pumila)

$6 / 7 \mathrm{ft}$.

$3.50 \quad 30.00$

$5 / 6 \mathrm{ft}$.

$3.00 \quad 25.00$

\section{CRAB-}

Carmine, (M. atrosanguinea)

Midget, (M. micromalus) shell pink

Scheidecker (M. scheideckeri)

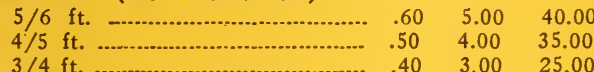

Flowering Bechtels (Malus)

Parkman (Parkmanii)

Sargent (Sargenti)

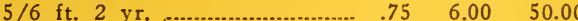

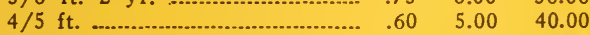

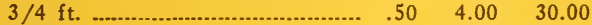

$2 / 3 \mathrm{ft} . \quad 40 \quad 3.00 \quad 25.00$

\section{DOGWOOD-}

Pacific, (Cornus nutalli)

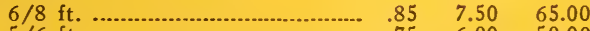

Mas, Cornelian Cherry (Cornus

Mascula)

$3 / 4 \mathrm{ft}$

Florida, (Cornus florida)

$2 / 3$ ft. .............................................. 60

Red Flower, (Cornus florida)

$18 / 24$ in. B. \& B........................ $2.00 \quad 17.50$

\section{ELM-}

American, (Ulmus americana)

\begin{tabular}{|c|c|c|c|c|}
\hline $12 \mathrm{ft}$. & .... & 1.00 & 8.50 & 75.00 \\
\hline $8 / 10 \mathrm{ft}$ & 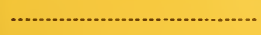 & .75 & 6.50 & 50.00 \\
\hline $6 / 8 \mathrm{ft}$ & 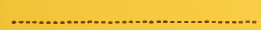 & .60 & 5.00 & 40.00 \\
\hline
\end{tabular}

Chinese or Siberian, (Ulmus

pumila)

$8 / 10 \mathrm{ft}$

$6 / 8 \mathrm{ft} .-80-7.50$

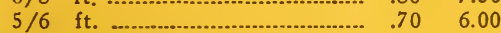

\section{GOLDENCHAIN-}

Laburnum (L. Vulgare)

$6 / 8 \mathrm{ft}$.

$5 / 6 \mathrm{ft}$.

$4 / 5 \mathrm{ft}$.

75.00

50.00

40.00

\section{HAWTHORN-}

Paul Dble. Scarlet, (Crataegus Each Per 10 per 100 oxycantha)

$7 / 8$ ft. 2 yr. branched................ $1.25 \quad 10.00 \quad 75.00$

$6 / 7$ ft. 2 yr. branched.............. $1.00 \quad 7.50 \quad 60.00$

$5 / 6$ ft. 2 yr. brancched............... $\quad .75 \quad 6.00 \quad 50.00$

$6 / 8$ ft. 1 yr. whips ................. $.75 \quad 6.00 \quad 50.00$

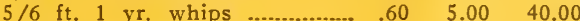

$4 / 5 \mathrm{ft} .1 \mathrm{yr}$. whips ............... $.50 \quad 4.00 \quad 30.00$

\section{HORSECHESTNUT-}

Aesculus White, (hippocastanum)

\begin{tabular}{|c|c|c|}
\hline$/ 8 \mathrm{ft} . \cdots$ & $\begin{array}{r}1.23 \\
.80\end{array}$ & $\begin{array}{r}10.00 \\
7.50\end{array}$ \\
\hline$/ 5$ ft. & .7 & 6.50 \\
\hline & & 5.5 \\
\hline
\end{tabular}

\section{LINDEN-}

American, (Tilia americana)

European (T. cordifolia)

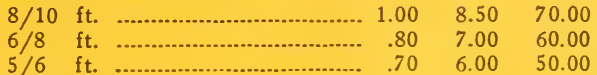

\section{LOCUST -}

Common Black, (Robinia pseudoacacia)

$8 / 10$ ft. $\quad \begin{array}{llll}.50 & 4.00 & 30.00\end{array}$

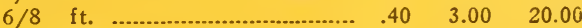

Honey Locust, (Gleditsia

triacanthas)

$6 / 8$ ft. ................................... $1.00 \quad 7.50$

$5 / 6$ ft. ..............................................

Rose Acacia, (Robinia hispida

macrophylla)

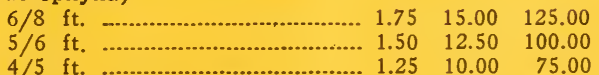

\section{MAPLE-}

Norway, (Acer plantanoides)

$8 / 10$ ft. 2 year headed and

branched heavy ....................

branched medium ............... $1.00 \quad 9.00 \quad 80.00$

$7 / 8$ ft. 2 year headed and

branched

$\begin{array}{llllll}8 / 10 & \text { ft. } 1 \text { year whips ….......... } & .70 & 6.00 & 50.00\end{array}$

$6 / 8$ ft. 1 year whips ................ $.65 \quad 5.50 \quad 45.00$

$5 / 6 \mathrm{ft} .1$ year whips ............. $\quad .40 \quad 3.00 \quad 25.00$

Schwedler, (A. pl. schwedleri)

$9 / 10$ ft. 2 year headed and

branched ............................ $1.75 \quad 15.00 \quad 125.00$

$7 / 8$ ft. 2 year headed and

branched medium ......... $1.50 \quad 12.50 \quad 100.00$

$6 / 8$ ft. 2 year headed and

branched ................................ $1.25 \quad 10.00 \quad 80.00$

$8 / 9 \mathrm{ft.} 1$ year whips ............. $1.25 \quad 11.00 \quad 100.00$

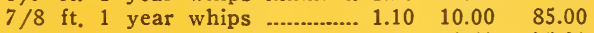

$6 / 7$ ft. 1 year whips ................. $1.00 \quad 8.50 \quad 75.00$

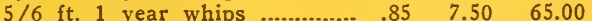

Sycamore (Platanus atropurpurea)

$8 / 10$ ft. .................................. $1.00 \quad 8.00 \quad 75.00$

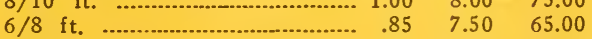

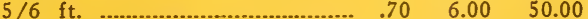

Silver, (Acer dasycarpum)

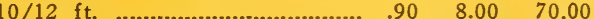

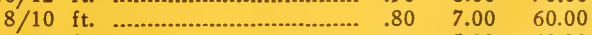

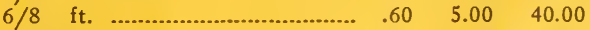

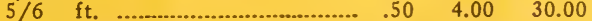

Oregon, (Acer macrophyllum)

$8 / 10$ ft. ................................. $\quad .80 \quad 7.00 \quad 60.00$

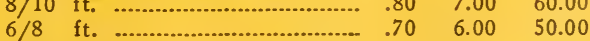

Vine Oregon, (Acer circinatum) $6 / 8 \mathrm{ft}$.

$5 / 6 \mathrm{ft}$

$1.00-8.50-75.00$

Japanese Red, Ribbon Leaf, (acer

palmatum atropurpureum)

$30 / 36$ in.

$18 / 24$ in 
Japanese Red, Threadleaf Wpg.,

(A. palmatum atro. dissectum) Each Per 10 Per 100

$24 / 30$ in. spread standards...... 4.00 35.00

$18 / 24$ in. spread standards...... $3.50 \quad 27.50$

$15 / 18$ in. spread standards...... 2.7522 .50

Japanese Green-leaf

$5 / 6$ ft. ........................................ $1.00 \quad 7.50$

\section{MULBERRY-}

Russian, (Morus alba tartarica)

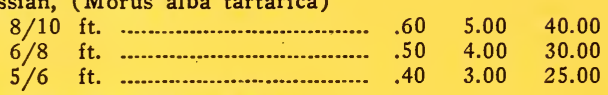

Teas Weeping, (Morus alba pendula)

2 yr. heads

$2.00 \quad 17.50$

1 yr. heads

$1.75 \quad 15.00$

\section{OAK-}

White, (Quercus alba)

Red, (Quercus rubra)

\begin{tabular}{|c|c|}
\hline $8 / 10$ ft. & 12.50 \\
\hline $6 / 8$ ft. & 10.00 \\
\hline $5 / 6$ ft. & 8.50 \\
\hline
\end{tabular}

\section{PEACH-}

Dble. Red (A. rubroplena)

Dble. Pink (A. roseaplena)

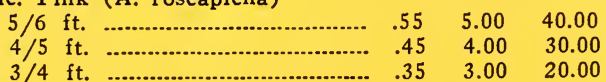

\section{PLANETREE-}

(Platanus Orientalis) branched

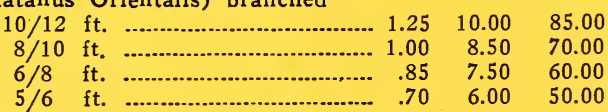

\section{PLUM-}

Prunus, Bliriana Dble. Pink Flg.

Thundercloud dark purple leaf,

Pissardi, purple leaf

Vesuvius, deep purple leaf

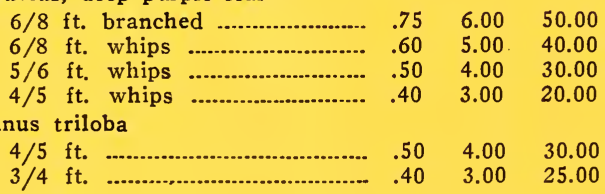

\section{POPLAR}

Carolina, (Populus eugenei)

Canadian, (P. deltoides)

Lombardy, (P. nigra italica)

$10 / 12 \mathrm{ft}$

$8 / 10 \mathrm{ft}$

Bolleana, (P. bolleana)

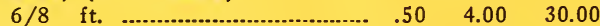

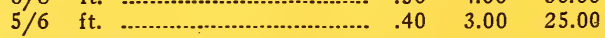

\section{SWEET GUM-}

(Liquidambar styraciflus)

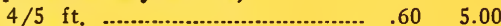

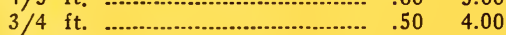

\section{TULIP TREE-}

(Liriodendron tulipifera)

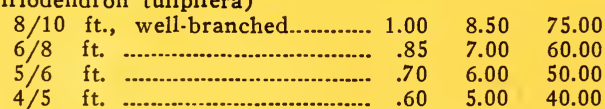

\section{WILLOW-}

Weeping Babylon (Salix babylonica) $8 / 10 \mathrm{ft}$.

$6 / 8 \mathrm{ft}$.
Weeping Wisconsin, (Salix blanda)

Each Per 10 Per 100

$6 / 8 \mathrm{ft}$.

$\begin{array}{lll}70 & 6.00 \quad 56.00\end{array}$

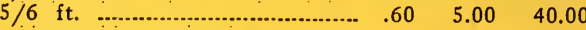

Russian Golden, (Salix Bab. aurea)

$8 / 10$ ft.

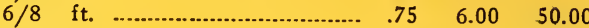

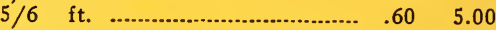

\section{Deciduous Flowering Shrubs}

\section{ALMOND-}

Pink or White Flg. (Prunus

glandulosa)

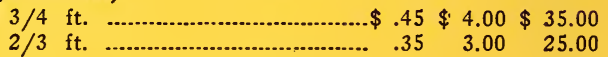

\section{ALTHEA-}

(Hibiscus syriacus)

$3 / 4 \mathrm{ft}$.

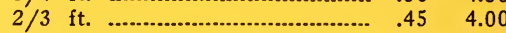

$18 / 24$ in. ..................................... .40 3.50

Boule de Feu, Red

Lady Stanley, Rosy White

Paeniflora, Rosy Purple

\section{AZALEA-}

Altaclare, (A. altaclarensis)

$18 / 24$ in., in bud .................... 1.7515 .00

$15 / 18$ in., in bud ....... $1.50 \quad 12.50 \quad 100.00$

$12 / 15$ in., in bud ...................... $1.25 .10 .00 \quad 90.00$

$9 / 12$ in. ....... $1.00 \quad 7.50 \quad 60.00$

chinese Mollis (A. mollis)

$24 / 30$ in., in bud $2.00 \quad 17.50$

$18 / 24$ in., in bud .................... 1.7515 .00125 .00

$15 / 18$ in., in bud ....................... 1.5012 .50100 .00

$12 / 15$ in., in bud ................... $1.2510 .00 \quad 90.00$

$9 / 12$ in. $1.00 \quad 7.50 \quad 60.00$

Western, (Occidentalis)

$24 / 30$ in., in bud ....................... 2.00 $17.50 \quad 150.00$

$18 / 24$ in., in bud ........................ $1.7515 .00 \quad 125.00$

$15 / 18$ in., in bud ................... $1.50 \quad 12.50 \quad 100.00$

$12 / 15$ in. $1.25 \quad 10.00 \quad 90.00$

\section{BARBERRY -}

Japanese, (Berberis thunbergi)

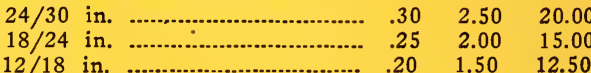

$12 / 18$ in. ........... $20 \quad 1.50 \quad 12.50$

New Red Leaf, (Berberis thun. atropurpureum)

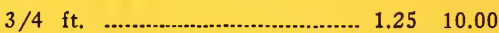

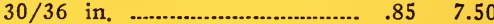

$24 / 30$ in. $\quad .70 \quad 6.00 \quad 50.00$

$18 / 24$ in. ................................................... 60 5.00

$15 / 18$ in. ................................... .55 $4.50 \quad 35.00$

$12 / 15$ in... $.50 \quad 4.00 \quad 30.00$

$9 / 15$ in. .................................... $40 \quad 3.00 \quad 25.00$

Wilson, (Berberis wilsoni)

$18 / 24$ in. ....................................... 6000

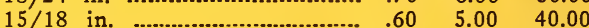

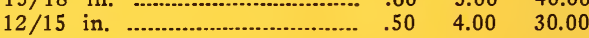

\section{BURNINGBUSH-}

(Euonymus europeus)

$\begin{array}{llll} & .50 & 4.00 & 35.00\end{array}$

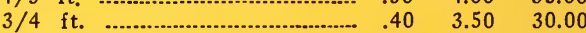

\section{BUTTERFLY BUSH-}

(Buddleia magnifica)

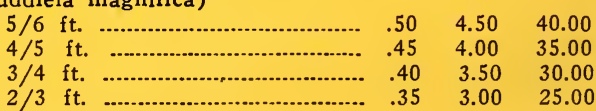

\section{CARYOPTERIS-}

Blue Spirea, late bloomer

$18 / 24$ in. 


\section{CEANOTHUS-}

(Marie Simon)

$30 / 36$ in. ................................ $1.25 \quad 10.00 \quad 85.00$ $24 / 30$ in.

Each Per 10 Per 100

CRANBERRY BUSH-

(Viburnum opulus)

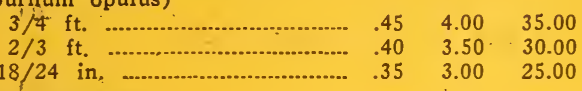

CORALBERRY -

(Symphoricarpos vulgaris)

$\begin{array}{llllll}3 / 4 & \mathrm{ft} . & \ldots & 25 & 2.00 & 15.00\end{array}$ $2 / 3 \mathrm{ft}$.

$.20 \quad 1.50 \quad 12.00$

\section{DEUTZIA-}

Lemoine, pink and white

$$
2 / 3 \mathrm{ft} \text {. }
$$

$18 / 24$ in.

Rose, Dble. scabra plena

$4 / 5 \mathrm{ft}$.

$3 / 4 \mathrm{ft}$.

$2 / 3$ fe.

Pride of Rochester

$4 / 5 \mathrm{ft}$.

$3 / 4 \mathrm{ft}$.

Gracilis, Pure white

$15 / 18$ in

$12 / 15$ in.

Fuzzy, scabra crenata alba

$4 / 5 \mathrm{ft}$.

$3 / 4 \mathrm{ft}$.

$2 / 3 \mathrm{ft}$.

Snowflake (D. candidissima)

$3 / 4 \mathrm{ft}$.

$2 / 3 \mathrm{ft}$.

\section{DOGWOOD-}

(Cornus elegantissima var.)

$2 / 3 \mathrm{ft}$.

$18 / 24$ in.

Siberian coral (Cornus sibirica)

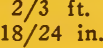

\section{ELDER-}

Cutleaf (Sambucus canadensis acutiloba)

Variegated (S. nigra var.)

$5 / 6 \mathrm{ft}$.

$\begin{array}{ll}4 / 5 & \mathrm{ft} . \\ 3 / 4 & \mathrm{ft} .\end{array}$

\section{FILBERT-}

Purple leaf

$3 / 4 \mathrm{ft}$.

.85

7.50

6.00

\section{FORSYTHIA-}

Border, (F. intermedia)

Fortune (F. suspensa fortunei),

Half upright

$4 / 5 \mathrm{ft}$.

$3 / 4 \mathrm{ft}$.

$2 / 3 \mathrm{ft}$.

Green stem, (F. viridissima)

$3 / 4 \mathrm{ft}$.

spensa Weeping (F. suspensa)

$4 / 5 \mathrm{ft}$.

$3 / 4 \mathrm{ft}$.

$2 / 3 \mathrm{ft}$.
$18 / 24$ in.

$\begin{array}{ll}.40 & 3.50 \\ .35 & 3.00\end{array}$

3.00
2.50

30.00

25.00

20.00

60.00

50.00

$$
18 / 24 \text { in }
$$

\section{HONEYSUCKLE}

Morrow, (L. morrowi)

Pink Tartarian, (L. tatarica)

White Tartarian, (L. tatarica alba)

$$
3 / 4 \mathrm{ft} \text {. }
$$

$2 / 3 \mathrm{ft}$.

$18 / 24$ in.

\section{HYDRANGEA-}

Otaksa, (H. opuloides) blue and pink

$18 / 24$ in

Each Per 10 Per 100

$15 / 18$ in

7.50

$12 / 15$ in.

$\begin{array}{ll}.70 & 6.00 \\ .60 & 5.00\end{array}$

Peegee (H. paniculata) Bush form $3 / 4 \mathrm{ft}$.

$2 / 3 \mathrm{ft}$.

$18 / 24$ in.

$$
.605 .00
$$

Peegee (H. paniculata)

Standards

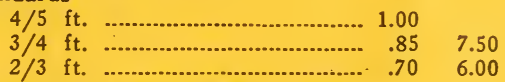

Smooth (H. Arborescens), Hills

of snow

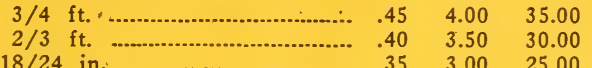

\section{OAK LEAF -}

\begin{tabular}{|c|c|}
\hline $\begin{array}{l}2 / 3 \mathrm{ft} . \\
18 / 24 \text { in. }\end{array}$ & .40 \\
\hline 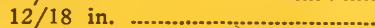 & 30 \\
\hline
\end{tabular}

(Hydrangea quercifolia) $15 / 18$ in.

40.00

\begin{tabular}{|c|c|c|c|}
\hline & & & \\
\hline 15 & .45 & 4.00 & \\
\hline $3 / 4 \mathrm{ft}$. & .40 & 3.50 & ( \\
\hline $2 / 3 \mathrm{ft}$. & .35 & 3.00 & \\
\hline
\end{tabular}

\section{KERRIA-}

Japanese Primrose, (K. Japonica)

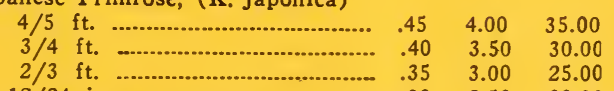

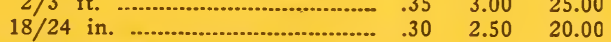

\begin{tabular}{|c|c|}
\hline 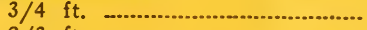 & .75 \\
\hline $18 / 24$ in. & $\begin{array}{l}.60 \\
.50\end{array}$ \\
\hline
\end{tabular}

Variegated, (K. japonica var.)

\section{LILACS-}

Common purple (Syringa vulgaris)

Common white

Persian Purple

$2 / 3$ ft. ......................................... $\quad .50 \quad 4.00$

Budded varieties

\section{MOCKORANGE}

(Philadelphus virginal)

$3 / 4 \mathrm{ft}$. ....................................... .45 4.00

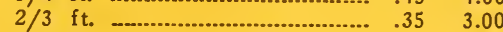

\begin{tabular}{|c|c|c|}
\hline ft. $\ldots$ & .50 & 4.50 \\
\hline $2 / 3$ ft. -............................................ & .45 & 4.00 \\
\hline
\end{tabular}

P. Gordon

P. Grandiflora

$4 / 5$ ft. ….............. $40 \quad 3.50 \quad 30.00$

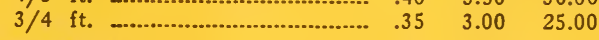

P. Avalanche

$3 / 4$ ft. .................................... $.35 \quad 3.00 \quad 25.00$

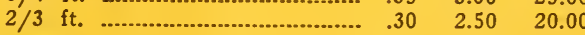

\section{QUINCE-}

Pink Flg., (Cydonia Japonica

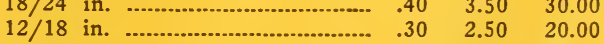

\section{SNOWBALL-}

Common, (Viburnum opulus sterilis)

$3 / 4$ ft. ..................................... $.60 \quad 5.00$

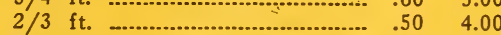

$18 / 24$ in. $40-3.50$

Fragrant (Viburnum carlesi)

$18 / 24$ in. ....................................... $1.50 \quad 12.50 \quad 100.00$

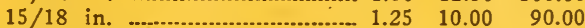

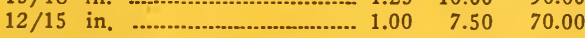

Japanese, (Vibrunum tomentosum

plicatum plenum)

$2 / 3 \mathrm{ft}$.

$.60 \quad 5.00$

$18 / 24$ in. 
SNOWBERRY-

Symphoricarpus racemosus

$3 / 4 \mathrm{ft}$.

$2 / 3 \mathrm{ft}$.

Each Per 10 Per 100

$\begin{array}{lll}30 & 2.50 & 20.00\end{array}$

SPIREA-

Anthony Waterer

$2 / 3 \mathrm{ft}$.
$18 / 24 \mathrm{in}$.

$15 / 18$ in.

Bridal Wreathe, (S. prunifolia)

$2 / 3 \mathrm{ft}$.

$18 / 24$ in.

$12 / 18$ in.

Garland, (S. arguta) white

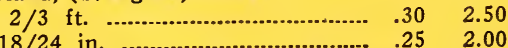

Japanese, pink and white (S. japonica)

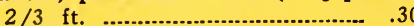
$18 / 24$ in $12 / 18$ in.

.25

Reeves, (S. reevesiana), white

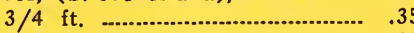
$2 / 3$ ft. .......................................

Van Houtte, (S. Van houttei) white $4 / 5 \mathrm{ft}$.

$3 / 4 \mathrm{ft}$.

Thunberg, (S. thunbergi,) pure white $3 / 4 \mathrm{ft}$.

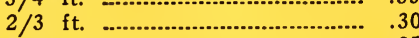
$18 / 24$ in $12 / 18$ in.

Golden Ninebark, (S. opulafolia aurea) $18 / 24$ in.

Japanese Pink, (S. japonica callosa rubra)

$2 / 3 \mathrm{ft}$.

$18 / 24$ in. $12 / 18$ in.

$\begin{array}{lll}.25 & 2.00 \quad 15.00\end{array}$

cean Spray, (Rock Spirea)

(Holodiscus discolor)

$2 / 3 \mathrm{ft}$.

\section{STRAWBERRY BUSH-}

(Euonymus americana) $3 / 4 \mathrm{ft}$

\section{TAMARIX-}

Africana pin

$\begin{array}{ll}4 / 5 & \mathrm{ft} . \\ 3 / 4 & \mathrm{ft} .\end{array}$

$2 / 3 \mathrm{ft}$.

\section{WEIGELA-}

Rosea, pink

$4 / 5 \mathrm{ft}$.

$2 / 3 \mathrm{ft}$.

Rosea variegated

$$
2 / 3 \mathrm{ft} \text {. }
$$

$18 / 24$ in.

Eva Rathke, red

$$
2 / 3 \mathrm{ft} \text {. }
$$

$$
18 / 24 \text { in. }
$$

Candida, white $2 / 3 \mathrm{ft}$.

$18 / 25$ in.

\section{Evergreens Conifers}

\begin{tabular}{|c|c|c|c|}
\hline$/ 7 \mathrm{ft}$. & 3.50 & 30.00 & 275.00 \\
\hline$/ 6$ & & 25.00 & 225.00 \\
\hline ft. & 2.25 & 20.00 & 175.00 \\
\hline 政。 & 1.50 & 12.50 & 100.00 \\
\hline 136 & 1.2 & 10.00 & 75.00 \\
\hline
\end{tabular}

\section{ARBORVITAE-}

American, (Thuja occidentalis)

$2.00 \quad 15.00$

$3.00 \quad 25.00$

$2.50 \quad 20.00$

$3.50 \quad 30.00$

$3.00 \quad 25.00$

$2.50 \quad 20.00$

$3.00 \quad 25.00$

$2.50 \quad 20.00$

$2.00 \quad 15.00$

$1.50 \quad 12.50$

$2.50 \quad 20.00$
American Globe, (Thuja occi-

dentalis globosa) Each Per 10 Per 100

$18 / 24$ in.

$\begin{array}{ll}1.50 & 12.50\end{array}$

$15 / 18$ in $\quad 1.00 \quad 8.50 \quad 75.00$

$12 / 15$ in. ….......................... $.85 \quad 7.50 \quad 65.00$

Berkmans Golden ( $T$. orien, aurea nana)

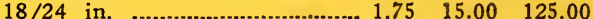

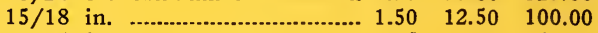

$12 / 15$ in. ................................. $1.25 \quad 10.00 \quad 90.00$

Golden (Thuja orientalis aurea)

$3 / 4 \mathrm{ft}$. ................................... $2.00 \quad 17.50 \quad 150.00$

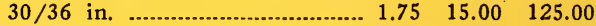

$24 / 30$ in. $\quad \begin{array}{llll}1.50 & 12.50 & 110.00\end{array}$

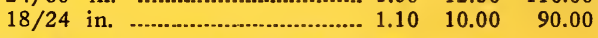

Golden Globe ( $T$. orien. Globe aurea)

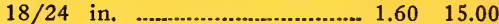

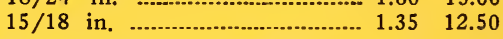

Golden Column, ( $\mathrm{T}$. orientalis

beverleyensis)

$4 / 5 \mathrm{ft}$.

$\begin{array}{lll}3.00 & 25.00 & 225.00\end{array}$

$1.50 \quad 12.50$

$30 / 36$ in

$\begin{array}{lll}2.50 & 20.00 & 175.00\end{array}$

$\begin{array}{lll}1.25 & 10.00 & 90.00\end{array}$

Goldspot (T. occ. aureavariegata)

$5 / 6$ ft. .......................................... $3.00 \quad 25.00$

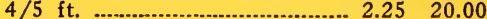

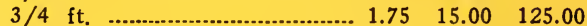

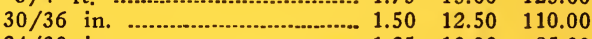

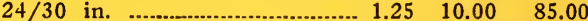

$18 / 24$ in. ...................................... $1.00 \quad 7.50 \quad 60.00$

Hovey ( $\mathrm{T}$. occ. hoveyi)

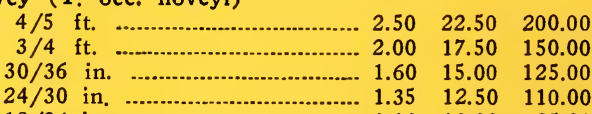

24/30 in.

Oriental (Thuja orientalis)

Oriental Pyramidal (T. or. pyramidalis)

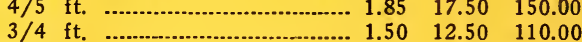
$30 / 36$ in. ................................ $1.25 \quad 10.00 \quad 85.00$ $24 / 30$ in. .................................. $1.00 \quad 7.50 \quad 60.00$

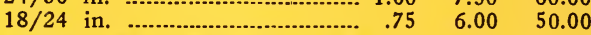

Oriental Compact (T. or. compacta)

$30 / 36$ in. ...................................... $1.75 \quad 15.00 \quad 125.00$

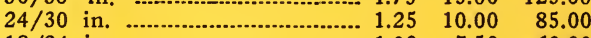

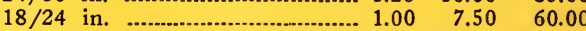

Pyramidal, American (T. occ. pyramidalis) $4 / 5$ ft. .......................................... $22.50 \quad 200.00$

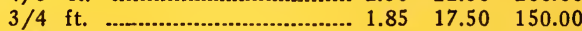
$30 / 36$ in $\quad \begin{array}{llll}1.60 & 15.00 & 125.00\end{array}$ $24 / 30$ in. ................................ $1.35 \quad 12.50 \quad 110.00$

Rosedale (T. or. rosedale)

$18 / 24$ in. ........................................ $1.50 \quad 12.50 \quad 100.00$

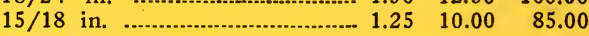

Ware (T. occ. wareana) $3 / 4$ ft. .......................................... $25 \quad 20.00$

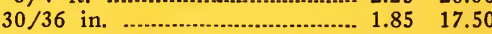

White Tipped ( $T$. occ. spicata alba) $15 / 18$ in. ...................................... $1.50 \quad 12.50 \quad 100.00$

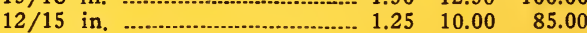

\section{ARBORVITAE-}

Woodward (T. occ. woodwardi)
$18 / 24$ in
$\begin{array}{lll}1.60 & 15.00 & 125.00\end{array}$

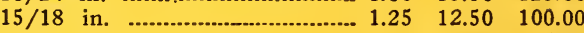

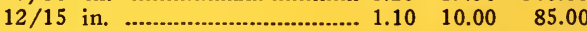
$10 / 12$ in. ... $90 \quad 8.00 \quad 70.00$

\section{CEDAR-}

Atlas, (Cedrus atlantica)

Deodar, (Cedrus deodara)

Lebanon, (Cedrus libani)

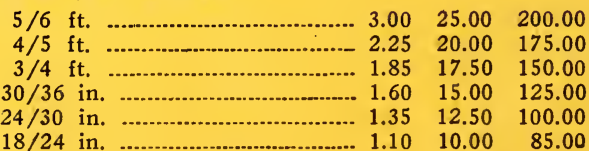




\section{CRYPTOMERIA -}

Japanese Plume, (C. japonica elegans)

Plume (C. japonica elegans) E,ach Per 10 Per 100

$5 / 6 \mathrm{ft}$.

$4 / 5 \mathrm{ft}$.

$3 / 4 \mathrm{ft}$.

$30 / 36$ in.

$24 / 30$ in

$18 / 24$ in.

$\begin{array}{lll}2.25 & 20.00 & 175.00\end{array}$

$\begin{array}{lll}2.00 & 17.50 & 150.00\end{array}$

$\begin{array}{lll}1.75 & 15.00 & 125.00\end{array}$

$\begin{array}{lll}1.50 & 12.50 & 100.00\end{array}$

$\begin{array}{rrr}1.25 & 10.00 & 85.00\end{array}$

$\begin{array}{lll}1.00 & 7.50 \quad 60.00\end{array}$

\section{CYPRESS-}

Arizona (Cupressus arizonica)

Italian (Cupressus sempervirens)

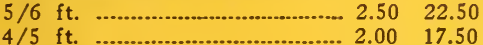

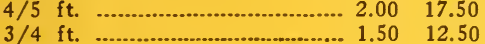

Hinoki (Chamaecyparis obtusa)

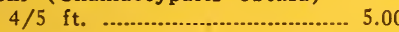

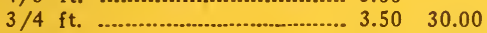

$30 / 36$ in. ............................... $2.50 \quad 20.00$

Hinoki Golden, (Cham. obtusa

a urea)

$4 / 5$ ft. .................................. 5.00

$3 / 4 \mathrm{ft}$. ............................ $3.50 \quad 30.00$

$30 / 36$ in 2.50

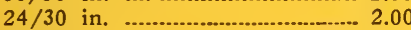

$18 / 24$ in. ................................ $1.75 \quad 15.00$

Hinoki Dwarf Golden

(Cham. obtusa aurea nana)

$18 / 24$ in. $2.00 \quad 17.50$

$15 / 18$ in. ...................................... 1.6015 .00

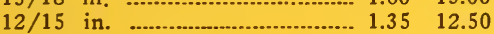

Lawson (Chamaecyparis lawsoni-

ana)

$6 / 8 \mathrm{ft}$.

$5 / 6 \mathrm{ft}$.

$4 / 5 \mathrm{ft}$.

$3 / 4 \mathrm{ft}$.

$30 / 36$ in

Green Column (C. law. Erecta

viridis)

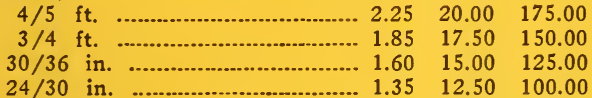

$\begin{array}{llll}1.65 & 12.50 & 100.00\end{array}$

$18 / 24$ in. ................................ $1.10 \quad 10.00 \quad 85.00$

Lawson Golden, (Cham. lawsoniana

lutea)

$30 / 36$ in.

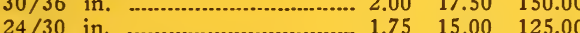

$18 / 24$ in $\quad 1.50 \quad 12.50 \quad 100.00$

Alumi (Cham. laws. alumi)

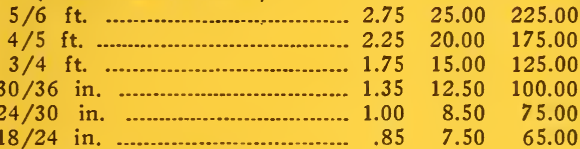

Silver Queen, (Cham. L.

lutescens )

$24 / 30$ in. ........................................... $2.00 \quad 17.50$

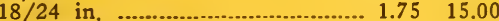

Triomphe de Boskoop

\begin{tabular}{|c|c|c|c|}
\hline$/ 6$ ft. & 3.50 & 30.00 & 250.00 \\
\hline $4 / 5$ ft. & 2.50 & 22.50 & 200.00 \\
\hline$/ 4 \mathrm{ft}$. & 1.85 & 17.50 & 150.00 \\
\hline & 1.60 & 15.00 & 1 \\
\hline & & 30.00 & 27 \\
\hline & & 20.00 & 5.0 \\
\hline & & $\begin{array}{l}17.50 \\
12.50\end{array}$ & \\
\hline
\end{tabular}

\section{FALSE ARBORVITAE-}

(Thujopsis dolobrata)

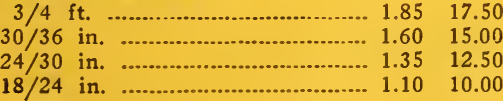

FIR

Douglas, (Pseudotsuga douglasi) Each Per 10 Per 100 $\begin{array}{llllll}3 / 4 & \mathrm{ft} & 1.50 & 12.50 & 100.00\end{array}$ $30 / 36$ in. ................................ $1.25 \quad 10.00 \quad 85.00$

White, (Abies concolor)

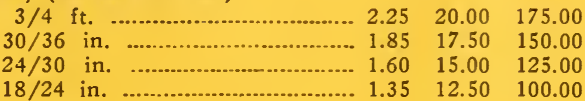

\section{HEMLOCK-}

Mountain ( $\mathrm{T}$ suga mertensiana)

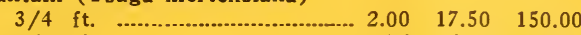

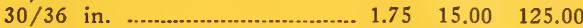

$24 / 30$ in. $1.50 \quad 12.50 \quad 100.00$

Canadian (Tsuga canadensis)

$3 / 4 \mathrm{ft}$. ........................................... $2.00 \quad 17.50 \quad 150.00$ $30 / 36$ in. $1.75 \quad 15.00 \quad 125.00$

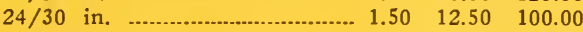

\section{JUNIPER-}

Chinese Creeping,

(Juniperus chinensis procumbens)

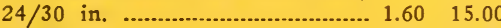

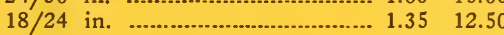

$15 / 18$ in. .................................. 1.2510 .00

Irish, Common (J. hibernica)

Irish, Pyramidal (J. hib. fastigiata)

$\begin{array}{crrrr}5 / 6 & \mathrm{ft} . \\ 4 / 5 \mathrm{ft} . \ldots \ldots \ldots \ldots \ldots \ldots \ldots\end{array}$

itzer (J. ch. pfitzeriana)

Savin (J. sabina)

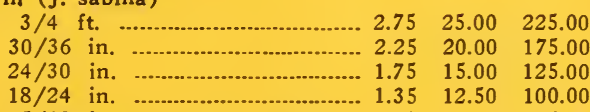

$15 / 18$ in. $1.10 \quad 10.00 \quad 85.00$

Pfitzer variegated, (J. chi. Pfitzeri-

ana varigata)

$30 / 36$ in. .......................................... 25.00

$24 / 30$ in. .................................... $1.85 \quad 17.50$

$18 / 24$ in. $1.60 \quad 15.00$

$15 / 18$ in. ..................................... 1.3512 .50

Redcedar, (Juniperus virginiana)

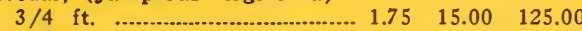

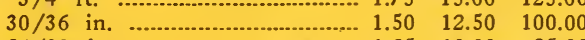

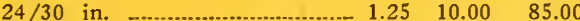

$18 / 24$ in. ..................................... $1.00 \quad 8.50 \quad 75.00$

Spiny Greek (J. erecta stricta)

$\begin{array}{lllll}3 / 4 & \mathrm{ft} & 2.25 & 20.00 & 175.00\end{array}$

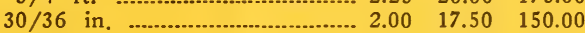

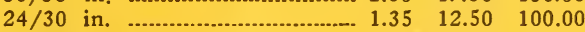

$18 / 24$ in $\quad 1.10 \quad 10.00 \quad 90.00$

Swedish ( 3 . suesica)

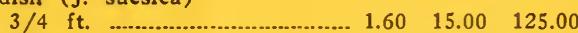

$30 / 36$ in. $\quad 1.35 \quad 12.50 \quad 100.00$

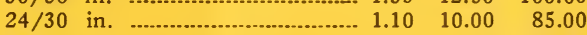

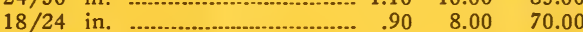

Tamarix Savin (J. sab.

tamariscifolia)

$18 / 24$ in. ...................................... 1.6015 .00

$15 / 18$ in. ................................. 1.3512 .50

\section{MONKEY PUZZLE-}

(Araucaria imbricata)

$18 / 24$ in.

$\begin{array}{ll}1.75 & 15.00\end{array}$

$15 / 18$ in $1.50 \quad 12.50$

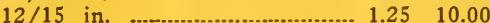

\section{PINE-}

Mugho

$18 / 24$ in. ...................................... $1.75 \quad 15.00 \quad 125.00$

$15 / 18$ in. ................................. $1.50 \quad 12.50 \quad 100.00$

$12 / 15$ in. $\quad 1.25 \quad 10.00 \quad 85.00$ 


\section{RETINOSPORA-}

Moss (Chamaecyparis pisifera squarrosa)

$6 / 7 \mathrm{ft}$.

$4 / 5 \mathrm{ft}$.

$3 / 4 \mathrm{ft}$.

$30 / 36$ in.

$24 / 30$ in.

$18 / 24$ in:

oss, Dwarf (Cham. p. sq. veitchi)

$24 / 30$ in. ..................................... 1.7515 .00

$18 / 24$ in. ….............................. $1.50 \quad 12.50$

$15 \% 18$ in ................... $1.25 \quad 10.00$

Plumo (Cham. p. plumosa)

Plume, Golden (Cham. p. pl. aurea)

$5 / 6 \mathrm{ft}$.

$4 / 5 \mathrm{ft}$

$\begin{array}{lllll}\text { ft. } & 1.75 & 15.00 & 125.00\end{array}$

$\begin{array}{lllll}30 / 36 & \text { in } & 1.50 & 12.50 & 100.00\end{array}$

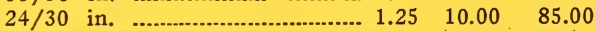

$18 / 24$ in. …................................ $1.00 \quad 8.50,75.00$

Sawara (Cham. pisifera)

$6 / 7$ ft. .............................. $2.50 \quad 25.00 \quad 225.00$

5/6 ft. .................................... $2.25 \quad 20.00 \quad 175.00$

$\begin{array}{lllll}4 / 5 & \mathrm{ft} . & 1.85 & 17.50 & 150.00\end{array}$

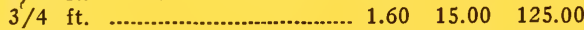

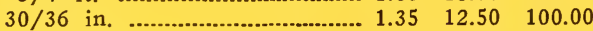

$\begin{array}{llll} & 1.10 & 10.00 & 85.00\end{array}$

Sawara Golden (Cham. p. aurea)

$4 / 5$ ft. ............................................ $25 \quad 20.00 \quad 175.00$

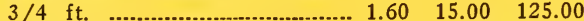

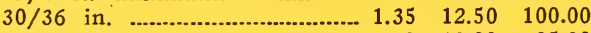

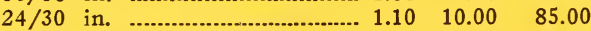

$18 / 24$ in. $\quad 90 \quad 8.00 \quad 75.00$

Thread (Ret. filifera)

$24 / 30$ in. ........................ $1.85 \quad 17.50$

$18 / 24$ in. $-1.60 \quad 15.00$

$15 / 18$ in.

$12 / 15$ in. ............................... $1.10 \quad 10.00$

Col. Blue (Picea pungens glauca)

(selected blue sdlgs.)

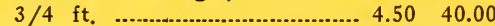

$30 / 36$ in. .................................... $3.75 \quad 35.00$

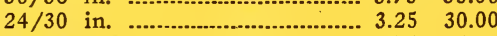

$18 / 24$ in. ....................................... $2.75 \quad 25.00$

$15 / 18$ in. $2.25 \quad 20.00 \quad 175.00$

Engelmann (Picea engelmanni)

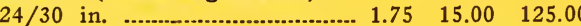

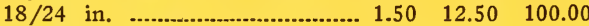

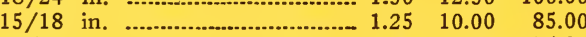

Kosters

$3 / 4$ ft. .................................... $8.50 \quad 70.00 \quad 500.00$

Norway, (Picea excelsa)

$5 / 6$ ft. ................................ $2.50 \quad 22.50 \quad 200.00$

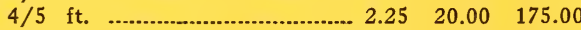

$3 / 4 \mathrm{ft} . \quad 1.75 \quad 15.00 \quad 125.00$

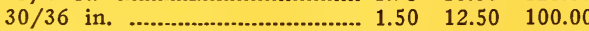

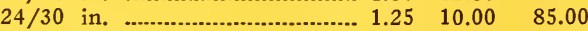

$18 / 24$ in. $1.00 \quad 9.00 \quad 75.00$

\section{YEW -}

Canadian, (Taxus canadensis)

$24 / 30$ in. ........................................... $2.00 \quad 17.50$

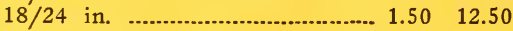

English, Erect or Prostrate types

(Taxus baccata)

$3 / 4 \mathrm{ft}$.

$30 / 36$ in

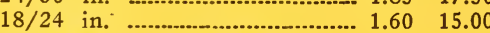

Irish, (Taxus baccata fastigiata)

$5 / 6 \mathrm{ft}$.

$4 / 5 \mathrm{ft}$

$3 / 4 \mathrm{ft}$.

$30 / 36$ in

$24 / 30$ in.

$18 / 24$ in
Irish Golden

$18 / 24$ in

Each Per 10 Per 100

$15 / 18$ in

$3.00 \quad 25.00$

\section{Broad Leaved Evergreens}

\section{ABELIA-}

(Abelia grandiflora)

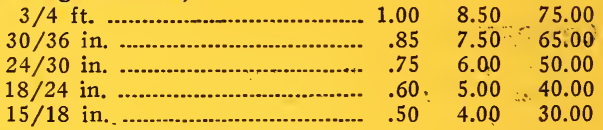

\section{ANDROMEDA-}

(Pieris japonica)

$9 / 12$ in.

$12 / 15$ in.

$15 / 18$ in

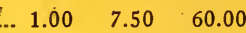

$18 / 24$ in.

90.00

100.00

\section{AUCUBA-}

Golddust (A. jap. var.)

Greenleaf (A. jap.)

$4 / 5 \mathrm{ft}$.

$3 / 4 \mathrm{ft}$.

$30 / 36$ in

$24 / 30$ in.

$18 / 24$ in.

$15 / 18$ in

$\begin{array}{ll}1.25 & 10.00\end{array}$

$\begin{array}{ll}1.50 & 12.50 \\ 1.75 & 15.00\end{array}$

$12 / 15$ in

\section{AZALEA-}

Hinodigiri

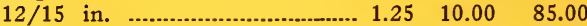

$10 / 12$ in $1.00 \cdot 8.50 \quad 75.00$

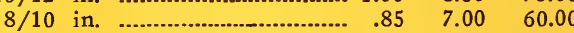

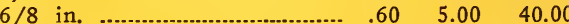

\section{BARBERRY-}

Darwin (Berberis darwini)

$18 / 24$ in. $\quad \begin{array}{llll}90 & 8.00 & 70.00\end{array}$

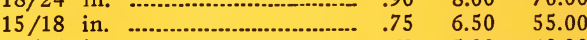

$12 / 15$ in. ...................................... $50 \quad 5.00 \quad 40.00$

Dulcis nana

Magellan, (Berberis buxifolia)

$\begin{array}{lllll}15 / 18 & \text { in. ... } & .90 & 8.00 & 70.00\end{array}$

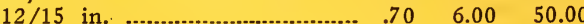

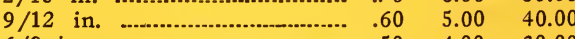

$6 / 9$ in. ........................... 4000

Rosemary Barberry (B. Stenophylla)

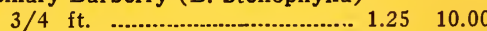

$30 / 36$ in. ...........................................

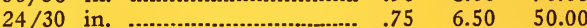

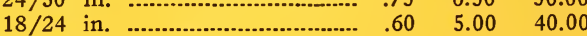

\section{BOX-}

Truetree, (Buxus sempervirens arborescens)

Pruned to pyramids or cones.

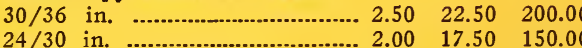

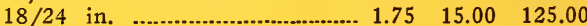
$15 / 18$ in. .................................. $1.50 \quad 12.50 \quad 100.00$

Pruned to globes.

$18 / 24$ in. $2.00 \quad 17.50 \quad 150.00$ $15 / 18$ in.

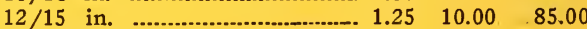

Not sheared.

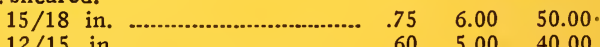

Truedwarf, Specimens (B. semp. suffruticosa)

$18 / 24$ in $\quad \begin{array}{llll}1.75 & 15.00 & 125.00\end{array}$

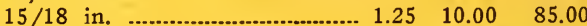

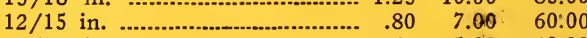

$10 / 12$ in. ..........................................

Silver (Buxus semp. argentea) $15 / 18$ in. ................................... $1.10 \quad 8.50$

$12 / 15$ in. ................................. 90 


\section{BROOM-}

Andreana, (Paradise Broom), Each Per 10 Per 100 (Cytisus andreanus)

Scotch, (Cytisus scoparius)

Daisy Hill

$$
2 / 3 \mathrm{ft} \text {. }
$$

Spanish (Genista hispanica)

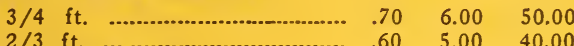

White (Cytisus albus)

$2 / 3 \mathrm{ft}$.

\section{CAMELLIA-}

Common Dble., (japonica) Red,

Pink and White

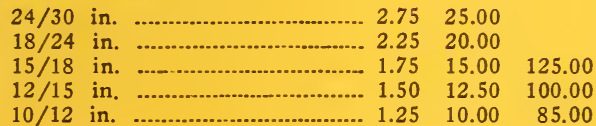

\section{COTONEASTER-}

\section{Applanata}

$4 / 5 \mathrm{ft}$.

$3 / 4 \mathrm{ft}$

Dielsiana

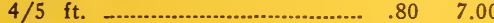

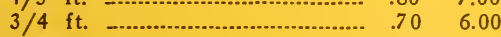

Franchetti

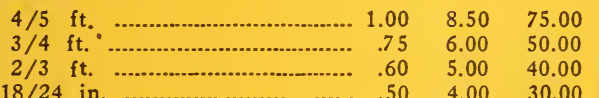

Harroviana

$$
2 / 3 \mathrm{ft} \text {. }
$$

Horizontalis

$$
3 / 4 \mathrm{ft} \text {. }
$$

$30 / 36$ in

$24 / 30$ in.

$18 / 24$ in.

$15 / 18$ in

Humifuca

$$
2 \text { in. pots }
$$

Microphylla

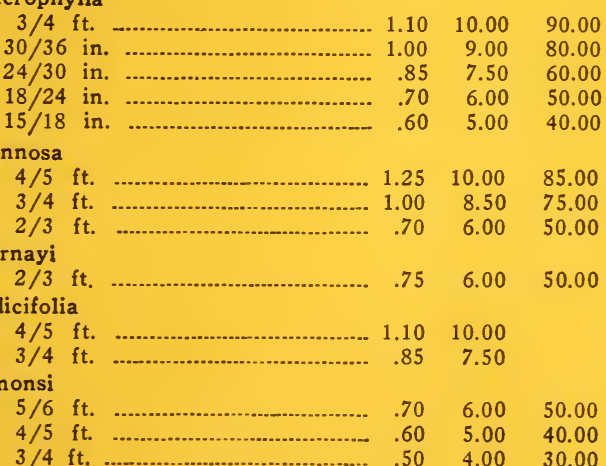

\section{DAPHNE-}

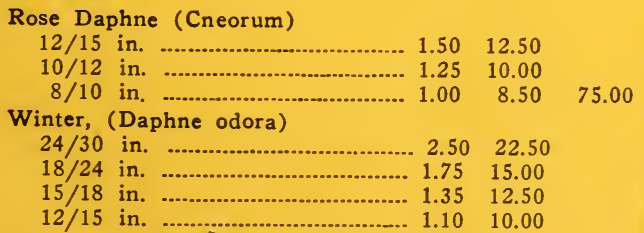

\section{ESCALLONIA-}

Red (Esc. rubra)

Rose (Est. rosea)

Langleyensis

White (Esc. berteriana)

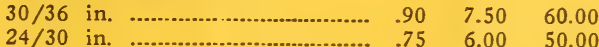

$24 / 30$ in. ........................................... 65 $6.00 \quad 50.00$

\section{EUONYMUS-}

Evergreen, (E. japonica)

$30 / 36$ in.

$18 / 24$ in. ....................................... $70 \quad 6.00 \quad 50.00$

$15 / 18$ in. ........................................... $50 \quad 5.00 \quad 40.00$

$12 / 15$ in. ....................................... $50 \quad 4.00 \quad 35.00$

Silver (E. jap. alba marginatus)

$15 / 18$ in. ........................... 50

$12 / 15$ in. $\ldots$

\section{FIRETHORN-}

Laland, (Pyracantha lalandi)

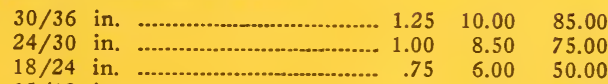

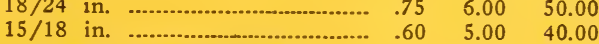

Narrow leaf, (P. augustifolia)

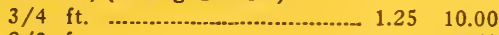

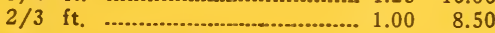

Scarlet, (P. coccinea)

$30 / 36$ in. .......................................... $1.25 \quad 10.00$

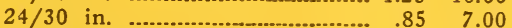

Yunnan (Pyr. yunnanensis)

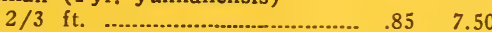

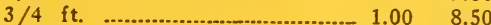

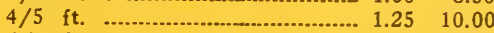

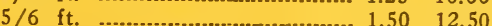

\section{HEATHER-}

Biscay (Erica mediterranea)

$24 / 30$ in. ........................................ 75

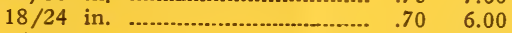

$15 / 18$ in. ............................................ 50

$12 / 15$ in. ............................................. $50 \quad 4.00 \quad 30.00$

Cornish (Erica vagans)

$15 / 18$ in. ............................................ $70 \quad 600$

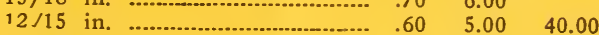

$9 / 12$ in. $\quad .50 \quad 3.50 \quad 30.00$

$6 / 9$ in. .......................................... $\quad .40 \quad 300$

Corsican (Erica stricta)

Tall Scotch (Calluna vulg. hammondi)

$18 / 24$ in. ................................... .60 $6.00 \quad 40.00$

$15 / 18$ in. ........................

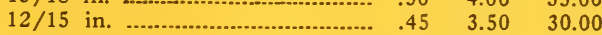

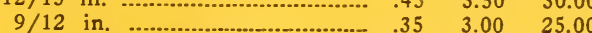

Darley (E. mediterranea hybrida)

Spring (E. carnea rosea)

$12 / 15$ in. ...................................... $50 \quad 5.000^{\circ} 40.00$

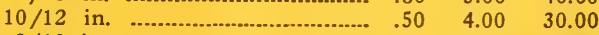

$8 / 10$ in. .................................................... 40

Irish Bell (Daboecia polifolia)

$2 / 3 \mathrm{ft}$. ..................................... 1.00

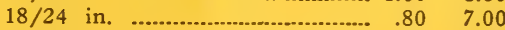

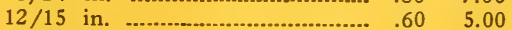

Scotch (Calluna vulgaris)

$18 / 24$ in.

$12 / 15$ in. ........................................ $50 \quad 4.50 \quad 40.00$

$10 / 12$ in. ............................................ 30 30

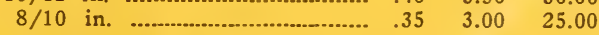

Swedish Bell (E. suecia)

$15 / 18$ in. ......................................... 70

$12 / 15$ in... $.60 \quad 5.00$

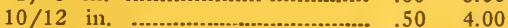




\section{HOLLY-}

English, (Ilex aquifolium.) Budded or grafted

$4 / 5 \mathrm{ft}$

$3 / 4 \mathrm{ft}$.

$30 / 36$ in

$24 / 30$ in.
$18 / 24$ in.

ariegated $24 / 30$ in.

Japanese (Ilex crenata) $24 / 30$ in.

$18 / 24$ in.

$15 / 18$ in.

HONEYSUCKLE-

(Lonicera nitida.) Evergreen

$24 / 30$ in.

$18 / 24$ in.

$15 / 18$ in.

Privet Honeysuckle (Lonicera

pileata)

$24 / 30$ in.

$18 / 24$ in.

$15 / 18$ in.

$12 / 15$ in.

\section{LAUREL-}

English, (Laurocerasus officinalis)

$30 / 36$ in.

$24 / 30$ in

$18 / 24$ in.

Portugal, Laurocerasus lusitanica

\begin{tabular}{|c|c|c|c|}
\hline$t / 5$ ft. $\ldots$ & 3.50 & 30.00 & \\
\hline $3 / 4$ ft. $\ldots . .$. & 2.50 & 22.50 & \\
\hline$/ 36$ in. ... & 2.00 & 17.50 & 160.00 \\
\hline $30 \mathrm{in.}$ & 1.60 & 13.50 & 120.00 \\
\hline in. & 1.25 & 10.00 & 90.00 \\
\hline i & 1.00 & 8.50 & 75.0 \\
\hline
\end{tabular}

\section{LAURISTINUS-}

\section{(Viburnum tinus)}

$30 / 36$ in.

$24 / 30$ in.

$18 / 24$ in.

$15 / 18$ in.

$12 / 15$ in.

Each Per 10 Per 100

$\begin{array}{ll}5.50 & 50.00\end{array}$

3.2530 .00

$2.75 \quad 25.00$

$2.25 \quad 20.00$

$3.00 \quad 25.00$

$2.00 \quad 17.50$

$\begin{array}{lll}1.75 & 15.00 & 125.00\end{array}$

$12.50 \quad 100.00$

$\begin{array}{ll}6.00 & 50.00 \\ 5.00 & 40.00 \\ 4.00 & 30.00 \\ & \\ 6.00 & 50.00 \\ 5.00 & 40.00 \\ 4.00 & 30.00 \\ 3.00 & 20.00\end{array}$

8.00

7.00

70.00

60.00

50.00

160.00

90.00

75.00

\section{MEXICAN ORANGE-}

\section{Choisya ternata}

$2 / 3 \mathrm{ft}$

$18 / 24$ in.

$1.35 \quad 12.50$ $15 / 18$ in.

$1.10 \quad 10.00$

\section{MYRTLE-}

Native of Southern Oregon

$18 / 24$ in.

$15 / 18$ in.

$12 / 15$ in.

1.10

$.90 \quad 8.00$

.807 .00

\section{MAGNOLIA -}

Sotithern (M. grandiflora)

$2 / 3 \mathrm{ft}$.

$3 / 4 \mathrm{ft}$.

\section{NANDINA DOMESTICA-}

$18 / 24$ in. $\begin{array}{ll}1.00 & 7.50\end{array}$ $12 / 18$ in.

$\begin{array}{ll}7.50 & 60.00\end{array}$ REGON GRAPE-

(Mahonia aquifolium.)

$18 / 24$ in.

$15 / 18$ in.

$12 / 15$ in.

$.75 \quad 6.00$

$.60 \quad 5.00$

.504 .00

50.00

40.00

PERNETTYA MUCRONATA-

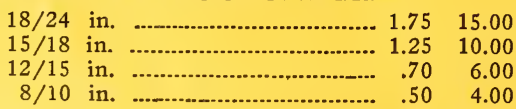

85.00

60.00

50.00

40.00

35.00

\section{PRIVET-}

California (Ligustrum ovalifolium)

Chinese. (L. sinense)

$4 / 5 \mathrm{ft}$.

$3 / 4 \mathrm{ft}$.

$2 / 3 \mathrm{ft}$. $\begin{array}{rr}4.00 & 35.00\end{array}$

$3.50 \ldots 30.00$

$18 / 24$ in.

$-3.00 \quad 25.00$

$2.00 \quad 15.00$

Glossy (L. lucidum)

Golden, (Ligustrum ovalifolium a urea)

$3 / 4$ ft. ............................................... $80 \quad 7.00 \quad 60.00$

$30 / 36$ in. ........................................... $70 \quad 6.00 \quad 50.00$

$24 / 30$ in. ........................................................

$18 / 24$ in. ............................................... 50

Japan, (Ligustrum japonica)

$30 / 36$ in. …..................................... $60 \quad 500 \quad 40.00$

$24 / 30$ in. $\quad 50 \quad 4.00 \quad 30.00$

$18 / 24$ in. .............................................. $30 \quad 3.00 \quad 25.00$

Lodense (L. nanum compactum)

$24 / 30$ in. ......................................... $30 \quad 2.25 \quad 20.00$

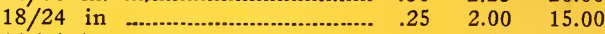

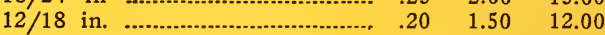

\section{RHODODENDRONS-}

Hybrid Seedling with buds

$24 / 30$ in. .... 3.0027 .50

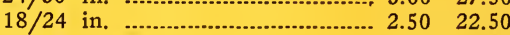

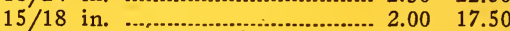

$12 / 15$ in. ................................... 1.5012 .50

Pink Pearl, with buds

Alice, with buds

$18 / 24$ in. ................................ $4.50 \quad 42.50$

$12 / 18$ in. .................................. $3.50 \quad 32.50$

Skimmia japonica

$12 / 15$ in. (Field) ..................... $1.25 \quad 10.00 \quad 85.00$

$9 / 12$ in. (5 in. pots) .............. $1.00 \quad 7.50 \quad 60.00$

\section{VERONICA-}

Buxifolia

Cupressoides

Erecta

Glaucafolia

$15 / 18$ in. ...................................... $40 \quad 4.50 \quad 40.00$

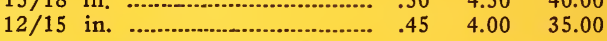

\section{VIBURNUM-}

Sweet (V, ordoratissimum)

$30 / 36$ in.

$24 / 30$ in

\section{Vines and Climbers}

\section{CLEMATIS-}

(Anemone.) Clematis montana

Sweet Autumn (Clematis paniculata)

3 yr. ..................................................

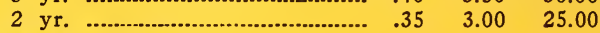

Montana, (Rubra)

2 yr.

$\begin{array}{lll}.30 & 2.50 & 20.00\end{array}$

$3 \mathrm{yr}$.

$\begin{array}{lll}.40 & 3.50 \quad 30.00\end{array}$

\section{CREEPER-}

Engelmann. (Ampelopsis quin-

quefolia engelmanni)

3 year _................................... . .30 $2.50 \quad 20.00$

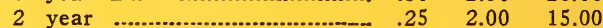

Boston Ivy. (Ampelopsis tricuspidata veitchii)

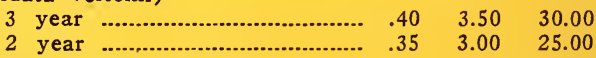

\section{DUTCHMANS PIPE-}

(Aristolochia sipho)

3 year

\section{HONEYSUCKLE-}

Belgian. (Lonicera periclymenum)

Hall's Japan. (Lonicera japonica halliana.)

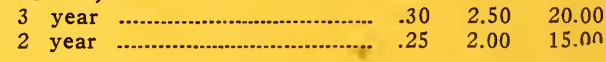




\section{IVY-}

English, (Hedera helix.)

2 year

\section{JASMINE-}

Red (Jasmimum beesianum)

White. (Jasminum officinale)

Winter (Jasminum nudiflorum)

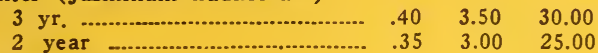

PERIWINKLE-

Common. (Vinca minor)

$$
3 \text { year }
$$

\section{TRUMPETCREEPER-}

(Bignonia radicans)

3 year .................................. .35 3.00

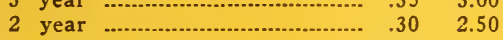

\section{WINTERCREEPER-}

Euonymus radicans, green and variegated

$15 / 18$ in

$12 / 15$ in.

$9 / 12$ in.

$6 / 9$ in.

Bigleaf, (Euonymus rad. vegetus)

$18 / 24$ in.

$15 / 18$ in -70

WISTERIA-

Chinese Purple. (Wisteria sinensis.) 3 year seedlings.

Chinese White, (Sinensis alba)

3 year seedlings
Each Fer 10 Per 100 $\begin{array}{lll}.25 & 2.00 & 15.00\end{array}$ $\begin{array}{lll}.25 & 2.00 & 15.00\end{array}$

$\begin{array}{lll}.75 & 6.00 & 50.00\end{array}$

$\begin{array}{lll}.60 & 5.00 & 40.00\end{array}$

$\begin{array}{lll}.45 & 4.00 & 30.00\end{array}$

$\begin{array}{lll}.35 & 3.00 & 25.00\end{array}$

$\begin{array}{ll}.70 & 6.00\end{array}$

$.60 \quad 5.00$
Longcluster, Purple. (Wisteria multijuga.)

3 year seedlings

3 year grafted

Each Per 10 Per 100 $\begin{array}{lrr}.45 & 4.00 & 35.00\end{array}$ $\begin{array}{lll}.75 & 6.50 & 50.00\end{array}$

\section{BOX-}

\section{Hedge Plants}

$\begin{array}{lllll}\text { Truedwarf. Buxus sempervirens } & 10 & 100 & 1000\end{array}$ suffruiticosa)

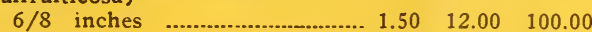

$4 / 6$ inches ..................................... $1.00 \quad 8.00 \quad 60.00$

LAUREL-

English. (Laurocerasus officin-

ales.)

$15 / 18$ in. ................................... 3.5030 .00

$12 / 15$ in. ....................................................... $300 \quad 25.00$

\section{OREGON GRAPE-}

(Mahonia aquifolium)

$6 / 8$ in.

PRIVET

$\begin{array}{ll}5.00 & 40.00 \\ 4.00 & 30.00\end{array}$

California. (Ligustrum ovalifolium.)

English. (Ligustrum 'vulgare)

1 and 2 year, $2-3$ feet.

1 and 2 year, 18-24 inches........

1 and 2 year, 12-18 inches

$4.00 \quad 35.00$

$3.50 \quad 30.00$

Lodense. Low branched. Dwarf habit, fine for borders or low hedge.

$12 / 15$ in. 

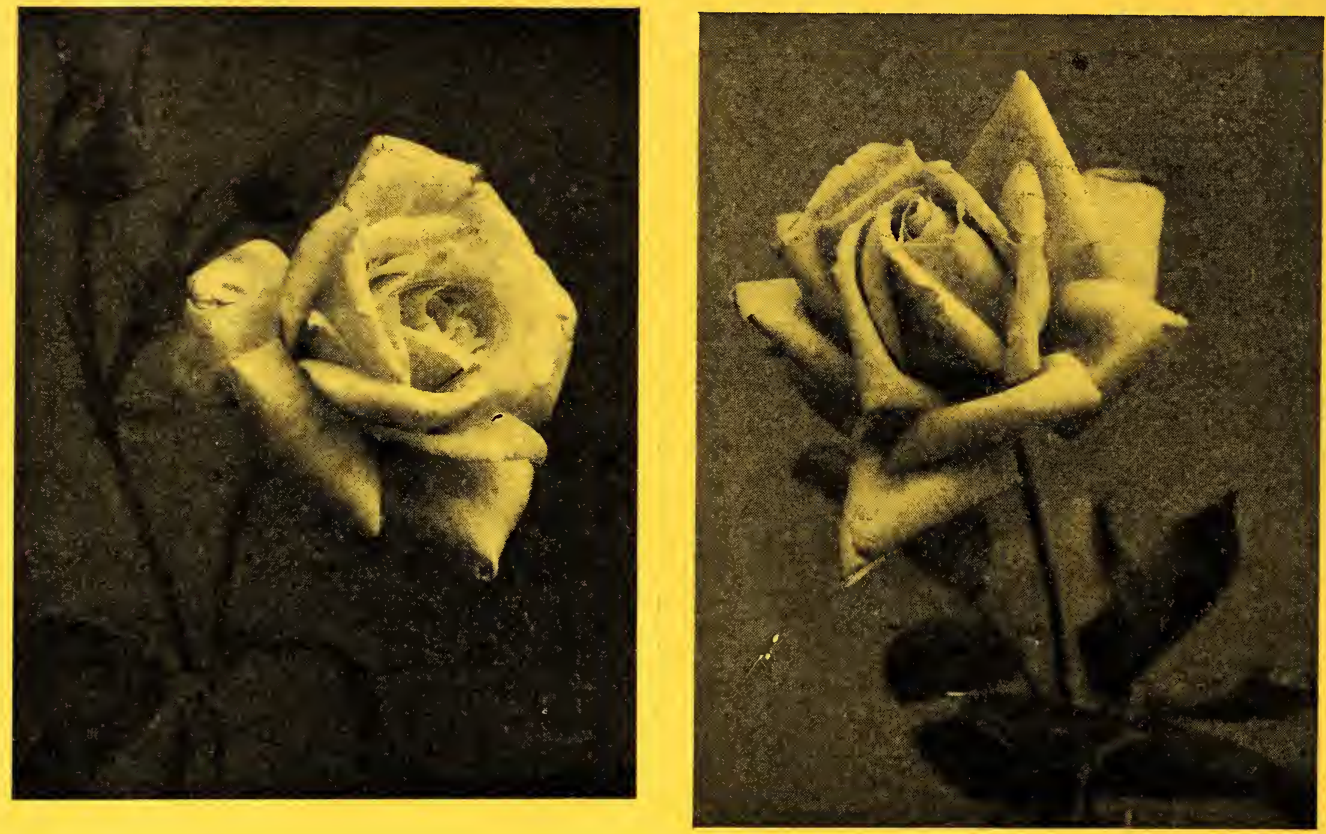

\section{Roses - Oregon Grown}

They are different. They are better.

The superior quality of our Oregon-grown field budded roses is now an established fact. For several years past the big eastern distributors have been using Oregon roses in car lots, coming back for more and more.

Our two year roses are grown and matured by the most approved methods. Irrigation is not practiced. Our Columbia River sandy loam soil, our ideal climatic conditions, and the proper cultural methods insure and produce the very best root system.

When grading we tie in bundles of ten. Orders should be for not less than five of a kind. We can and will break bundles to serve our patrons, but must charge extra for handling when numerous small lots are wanted. Make your order conform as nearly as possible to ten of a kind.

In our list you will find many new and rare varieties of recent introduction. A pleased eastern customer wrote us on March 14 this year: "These roses are the finest I have ever received in twenty-five years' experience with roses."

\begin{tabular}{|c|c|c|c|c|}
\hline & & & Per 10 & 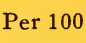 \\
\hline$\$ 3.00$ & $\$ 25.00$ & & 3.00 & \\
\hline 3.00 & 25.00 & land, old rose & 3.00 & \\
\hline kander Hill Gray, lemon-yellow ............ 3.00 & 25.00 & ch-pinl & & \\
\hline elia Gravereaux, dark red ...................... 3.00 & 25.00 & Constance, orange buds & 3.00 & 25 \\
\hline ca, rose-pink .................................. 3.00 & 25. & m, crimson & & \\
\hline can Beauty, pink ............................ 3.00 & & er, & 3.00 & \\
\hline -pink (...................... 3.00 & & rl & & \\
\hline t 3.50 & & pink & 3.50 & \\
\hline 3.00 & 25 & Edit & & \\
\hline & & ine & & \\
\hline & & & & \\
\hline Bo & & Do & 3.50 & \\
\hline (n) & & 20 & 3.50 & \\
\hline Bet & & $D_{c}$ & 3. & \\
\hline $\mathrm{Hul}$ & & 2 & 3. & \\
\hline Bet & & Du & 4. & 35 \\
\hline 3.00 & & 1-yellow & & \\
\hline 2.50 & & $\mathbf{E d}$ & 3.00 & 25 \\
\hline Cap & 2 & M. & & \\
\hline ............... 2.50 & 20. & Edith Nelli & 3. & 30.00 \\
\hline (n)................... & & Edi & & \\
\hline ......... 3.00 & 25. & E. & 4.00 & 35. \\
\hline (n......... 3. & & awley, crims & 3.00 & \\
\hline …......... 3.50 & & yellow $\ldots$ & 3.50 & \\
\hline 2.5 & & & & \\
\hline & & kcwith, r & 3.00 & \\
\hline
\end{tabular}


Per 10 Per 100

Elvira Aramayo, reddish-copper

Emma Wright, orange

3.50

Empire Queen, cerise

3.00

Etoile de Feu, orange pink .......................... 3.00

Etoile de France, crimson ............................... 3.00

Etoile de Hollande, scarlet ............................. 3.00

Felicity, pink ……............................................. 3.00

Feu Joseph Looymans, yellow ..................... 3.50

F. J. Grootendorst, carmine ........................... 3.00

Flame of Fire, orange .................................... 3.00

Francis Scott Key, crimson ......................... 3.00

Fragrant Bouuet, rose-pink ............................ 3.00

Frank Reader, yellow ........................ 3.00

Franklin, light salmon ....................................... 3.00

Frau Karl Druschki, white ............................ 2.50

Geisha, orange-yellow ......................................... 3.00

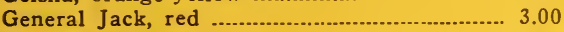

General McArthur, scarlet .............................. 2.50

General Sup. Janssen, carmine ...................... 2.50

George Arends, rose-pink ............................. 2.50

George C. Waud, light red ............................ 2.50

George Dickson, red .............................. 2.50

Gloire Ched. Guin, crimson .......................... 2.50

Golden Emblem, yellow .................................. 3.50

Golden Lady Pierrie, yellowish white ........ 3.50

Golden Ophelia, yellow ..............................- 3.00

Gorgeous, orange-yellow ………………............ 3.00

Grootendorst Pink, pink …_........................ 3.00

Grootendorst Red, red ................................. 3.00

Grange Colomb, creamy white ...................... 3.00

Gruss an Teplitz, crimson ....................... 2.50

Gwyneth Jones, shell-pink ............................ 3.00

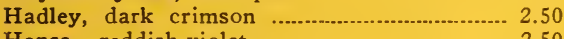

Hansa, reddish-violet .................................... 2.50

Harrison's Yellow, yellow ............................. 2.50

Hawlmark Crimson, scarlet .............................. 3.00

Hermosa, deep pink ........................................ 2.50

Holt Hewitt, velvety crimson ....................... 3.50

Hoosier Beauty, crimson-scarlet ...................... 3.00

Hortulaneous Budde, carmine …................... 3.00

Hortulaneous Fiet, ochre-yellow .................... 3.00

Hugh Dickson, brilliant scarlet ..................... 2.50

H. V. Machin, crimson ................................ 2... 20

Imperial Potentate, pink ................................ 3.00

Independence Day, orange ................................ 3.00

Irish Elegance, orange-pink ........................... 3.00

Irish Fireflame, crimson ................. 3.00

Irish Charity, scarlet ……............................ 3.50

Irish Hope, brilliant red .................................. 3.50

Isobel, crimson and orange ......................... 3.00

Ivy May, rose to amber .............................. 3.50

J. B. Clark, black maroon ............................. 2.50

Jean Forestier, carmine-orange …................... 3.00

J. J. L. Mock, cherry red .......................... 3.00

Joanna Hill, clear yellow ............................... 4.00

John Ruskin, red rugosa ................................ 3.00

John Russell, crimson ................................ 3.00

J. tto Thilow, rose-pink ................................... 3.00

Joseph Hill, salmon-pink ............................... 3.00

Juliet, gold and rose ........................................ 2.50

Jules Tarbart, coppery pink ............................... 3.50

Kaiserin, creamy-white .............................. 3.00

K. A. Victoria, snow-white ......................... 3.00

K. of K., velvety scarlet ............................... 3.00

Killarney Brilliant, crimson ............................. 2.50

Killarney Double White, white .................... 2.50

Killarney Queen, rosy-pink .......................... 2.50

La France, silvery-pink .................................. 3.00

Lady Alice Stanley, coral-rose ........................ 2.50

Lady Ashtown, deep pink ............................. 2.50

Lady Florence Strong, gold pink .................. 3.00

Lady Hillingdon, saffron-yellow .................... 3.00

Lady Inchiquin, orange-cerise ....................... 3.50

Lady Margaret Stewart, yellow ...................... 3.50

Lady Pierrie, apricot-pink ……........................ 3.00

Lady Roberts, apricot .................................. 3.00

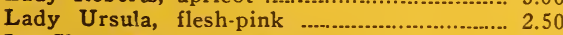

La Champagne, rose peach .............................. 2.00

Laurant Carle, carmine ..................................... 2.50

Legion, deep cerise red ...................................... 3.50
30.00

5.00

25.00

25.00

25.00

25.00

30.00

25.00

$(-000$

25.00
25.00

25.00

25.00

20.00

25.00

25.00

20.00

20.00

20.00

20.00

20.00

20.00

30.00

30.00

25.00

25.00

25.00

25.00

25.00

20.00

25.00
20.00

20.00
20.00

20.00

25.00

20.00

30.00

25.00

25.00

25.00

20.00

20.00

25.00

25.00

25.00

30.00

30.00

25.00

30.00

20.00

25.00

25.00

35.00

25.00

25.00

25.00

25.00

20.00

30.00

25.00

25.00

25.00

20.00

20.00

20.00

25.00

20.00

20.00

25.00

25.00

30.00

30.00

25.00

20.00

25.00

20.00
30.00
Per 10 Per 100

Lord Charlemont, crimson $3.50-30.00$

Lord Lambourne, buttercup-yellow .............. $3.50 \quad 30.00$

Los Angeles, flame-pink ............................... $3.00 \quad 25.00$

Louise C. Breslau, red .............. $3.00 \quad 25.00$

Lulu, orange pink to copper .......................... $3.00 \quad 25.00$

Mabel Lynnas, crimson with yellow ............. $3.50 \quad 30.00$

Mabel Morse, golden yellow …..................... $3.00 \quad 25.00$

Mme, Butterfly, apricot

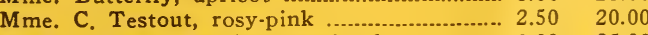

Mme. Edouard Herriot, coral-red ................. $3.00 \quad 25.00$

Mme. Jules Bouche, white ……...... $3.00 \quad 25.00$

Mme. Leon Paine, salmon-pink ...................... $3.00 \quad 25.00$

Mme. Marrerin, crimson scarlet ................... $3.00 \quad 25.00$

Mme. Melaine Soupert, yellow ..............-. $3.00 \quad 25.00$

Mme. Ravary, golden cream ............................ $3.00 \quad 25.00$

Mme. Ray Poincare, pale pink ….................... $3.00 \quad 25.00$

Mme, Segond Weber, rose-pink ................... $2.50 \quad 20.00$

Magna Charta, bright pink ............................ $2.50 \quad 20.00$

Naman Cochet, pink .................................... $3.00 \quad 25.00$

Maman Cochet, white ........ $3.00 \quad 25.00$

Marcia Stanhope, white ............................. $3.50 \quad 30.00$

Margaret de Andone, deep crimson .............. $6.00 \quad 50.00$

Nargaret McGredy, oriental red ................... $3.00 \quad 25.00$

Marian Cran, scarlet ...... $3.50 \quad 30.00$

Mary Pickford, orange-yellow ….................... $3.00 \quad 25.00$

Miss Cynthia Ford, pink ............................... $3.00 \quad 25.00$

Niss Lollita Armour, yellow ...................... $3.00 \quad 25.00$

Miss Mona Hunting, salmon …........................ $3.00 \quad 25.00$

Niss Rowena Thom, pink ........................... $3.00 \quad 25.00$

Miss Wilmot, soft cream $\quad 3.00 \quad 25.00$

Modesty, cream and rose ................................. $3.00 \quad 25.00$

Mons. Julien Potin, deep yellow ................ $3.00 \quad 25.00$

Moss, red ........... $3.00 \quad 25.00$

Mrs. Aaron Ward, indian yellow …................. $3.00 \quad 25.00$

Mrs. Alfred Tate, salmon …........................... $3.00 \quad 25.00$

Mrs, Ambrose Richardo, rosy yellow …...... $3.00 \quad 25.00$

Mrs. A. R. Barraclugh, pink ...................... $3.50 \quad 30.00$

Mrs. A. R. Waddell, scarlet .......................... $3.00 \quad 25.00$

Mrs. Beckwith, yellow buds …....................... $3.00 \quad 25.00$

Mrs. Chas. Bell, shell-pink …......................... $3.50 \quad 30.00$

Mrs. Chas. Lamplough, pink .......................... $3.00 \quad 25.00$

Mrs. Chas. Russell, pink ….......................... $3.00 \quad 25.00$

Mrs. Courtney Page, orange-cerise …........... $3.50 \quad 30.00$

Mrs. E. Pem. Thom., lemon-yellow ….......... $3.00 \quad 25.00$

Mrs. Farmer, indian yellow ......................... $3.00 \quad 25.00$

Mrs. F. R. Dennison, light pink ….......... $3.00 \quad 25.00$

Mrs. F. R. Peirson, crimson …..................... $3.00 \quad 25.00$

Mrs. G. A. Van Rossum .......... $4.00 \quad 35.00$

Mrs, R. W. Vanderbilt, red ......................... $3.00 \quad 25.00$

Mrs. Henry Morse, pink …............................. $3.00 \quad 25.00$

Mrs. Henry Bowles, rosy-pink ........................ $3.00 \quad 25.00$

Mrs. Herbert Nash, crimson .............. $3.50 \quad 30.00$

Mrs. Herbert Stephens, white …..................... $3.00 \quad 25.00$

Mirs. H. R. Darlington, pure-white ................. $3.00 \quad 25.00$

Mrs. James Heath

Mrs. John Laing, clear pink …..................... $3.00 \quad 25.00$

Mrs. Lovell Swisher, salmon-pink ................. $3.00 \quad 25.00$

Mrs. Redford, apricot-orange …................ $3.00 \quad 25.00$

Mrs. T. Smith, glowing cerise ........................ $3.50 \quad 30.00$

Mrs. W. C. Egan, rose-pink ............................. $3.00 \quad 25.00$

Mrs. W. C. Miller, bright pink ……….... $2.50 \quad 20.00$

Mrs. W. E. Nickerson, yellow ...................... $3.50 \quad 30.00$

Norman Lambert, copper-orange …................ $3.50 \quad 30.00$

Old Golde, coppery red ................................ $3.00 \quad 25.00$

Ophelia, light salmon …............-...... $3.00 \quad 25.00$

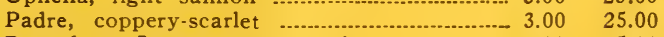

Partadown Ivory, creamy white …................. $4.00 \quad 35.00$

Patience, orange carmine $\ldots . . .50 \quad 3.00$

Paul Neyron, deep rose .................................... $3.00 \quad 25.00$

Pax Labor, yellow …-................................ $3.00 \quad 25.00$

Persian Yellow, golden yellow …........ $\quad 2.50 \quad 20.00$

Pharisear, silvery pink …....................................... $2.50 \quad 20.00$

Pilgrim. silvery pink ..................................... $3.00 \quad 25.00$

Pink Cochet, rose-pink …................ $3.00 \quad 25.00$

Pink Pearl, pink-rose …................................. $3.50 \quad 30.00$

Premier, dark pink .................................... $3.00 \quad 25.00$

President Cherieux, salmon pink .................. $3.00 \quad 25.00$

President Herbert Hoover, scarlet red........... $5.00 \quad 40.00$

President Jac Smits, scarlet ......................... $5.00 \quad 40.00$

Prince de Bulgarie, silvery-pink _... $\quad 3.00 \quad 25.00$

Prince C. Rohan, maroon ............................... 2.50 20.00

25.00 
Per 10 Per 100

Queen of Fragrance, shell pink 2.50

3.00

Radiance, red .................................................. 3.00

Rapture, dark pink .................................... 3.00

Red Letter Day, scarlet ........................... 3.00

Rev. F. Page Roberts, yellow ................... 3.50

Rose Hill, rosy pink .................................... 3.00

Roselandia, yellow ......................................... 3.00

Rose Marie, bright red ..................................... 3.00

Royal Red, scarlet ......................................... 3.50

Rugosa Alba, white, single .............-............. 3.00

Rugosa Anthony Waterer, red rugosa ........... 2.50

Rugosa Rubra, scarlet .............................. 3.00

Sarah Van Fleet, rose-pink ............................ 2.50

Scarlet Glory, scarlet -......................... 3.00

Sensation, scarlet-crimson …........................... 3.00

Shot Silk, coppery-rose .............................. 3.00

Soliden, carmine

Soliel d'Orr, orange to pink .......................... 3.00

Sir David Davis, crimson ................................ 3.00

Sunburst, deep apricot ................................... 3.00

Sunstar, orange-yellow .................-_-................ 3.00

Souv. de Cl. Pernet, orange-yellow .............. 3.00

Souv. de George Beckwith, salmon-pink ...... 3.00

Souv, de George Pernet, oriental-red .......... 3.00

Souv, de H. A. Verschuren, yellow .............. 3.00

Talisman, bright apricot ......................... 5.00

Templar, crimson ..- 3.00

Toison d'Orr, orange-yellow ........................... 3.00

Turk's Rugosa Samling, salmon-pink .............. 3.00

Ulricch Brunner, cherry-red .......................... 3.00

Una Wallace, old rose .................................... 3.00

Vesuvius, crimson

Ville de Paris, yellow ................................... 4.00

Viscountess Davenport, yellow .................... 3.00

Willomere, pink-coral ............................... 3.00

Wm. F. Dreer, orange-pink ...................... 3.00

Wilhelm Kordes, golden-salmon ...................... 3.50

W. R. Smith, creamy white .......................... 3.00

\section{Climbing Roses}

American Beauty, crimson

American Pillar, pink ................................... $2.50 \quad 20.00$

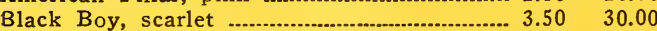

Cecil Brunner, rosy-pink ............................. $3.00 \quad 25.00$

Chaplin's Pink, dark pink ............................ $5.00 \quad 40.00$

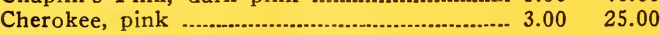

Crimson Rambler, crimson ....-...-........... $2.50 \quad 20.00$

Chateau, black ......................................... $3.00 \quad 25.00$

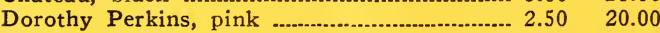

Dorothy Perkins, red ... $2.50 \quad 20.00$

Dorothy Perkins, white $\quad 2.50 \quad 20.00$

Dr. Van Fleet, flesh-pink .................................. $3.00 \quad 25.00$

Emily Gray, yellow ....................................... $3.50 \quad 30.00$
Per 10 Per 100

Eugene Jaquet, scarlet 25.00

$\begin{array}{lll}\text { Excelsa, dark red } & 2.50 & 20.00\end{array}$

Flower of Fairfield, crimson …........................... $2.50 \quad 20.00$

General McArthur, red ....................................... 2.0 $2.00 \quad 25.00$

Gloire de Dijon, yellow $3.00-25.00$

Gruss an Teplitz, scarlet …............................. $3.00 \quad 25.00$

Hadley, crimson .................................................. $3.00 \quad 25.00$

Hiawatha, crimson .................. $2.50 \quad 20.00$

Hoosier Beauty, crimson ............................... $3.00 \quad 25.00$

H. V. Machin, scarlet .................................... $3.00 \quad 25.00$

Jacotte, dark yellow ....................................... $2.50 \quad 20.00$

Kaiserin, white $\quad 3.00 \quad 25.00$

Lady Ashtown, pink ............................................... $3.00 \quad 25.00$

Lady Hillingdon, apricot ................................ $3.50 \quad 30.00$

Lemon Pillar, yellow $\quad 2.50-20.00$

Los Angeles, pink ................................................. 3.00

Louise Breslau, coral-red .............................. $3.00 \quad 25.00$

Mme. Alfred Carriere, white ..................... $3.00 \quad 25.00$

Mme. C. Testout, pink .................................. $2.50 \quad 20.00$

Mme. E. Herriot, orange ...................................... $3.00 \quad 20.00$

Marie Gauschalt, brilliant pink .................... $2.50 \quad 20.00$

Marschael Neil, yellow ................................ $3.50 \quad-30.00$

Miss Mary Wallace, pink ................................... $3.00 \quad 25.00$

Ophelia, apricot-pink ... $3.00 \quad 25.00$

Paul's Scarlet, scarlet …............................ $2.50 \quad 20.00$

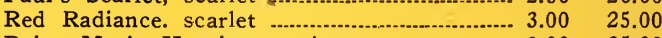

Reine Marie Henriette, crimson …............... $3.00 \quad 25.00$

Silvermoon, pure white $\quad 3.00 \quad 25.00$

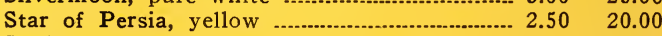

Sunburst, yellow ................................................. $3.50 \quad 30.00$

Tausendschoen, white to pink …................... $2.50 \quad 20.00$

Wichuriana Alba, white ............................................ $3.00 \quad 25.00$

Wm. A. Richardson, copper ......................... $3.00 \quad 25.00$

\section{Baby Roses}

Catherine Zeimet, white ... $2.50 \quad 20.00$

Cecil Brunner, pink ....................................... $3.50 \quad 30.00$

Clothilde Soupert, white ….........................-...... $2.50 \quad 20.00$

Chatillion, pink and orange .......................... 2.50 20.00

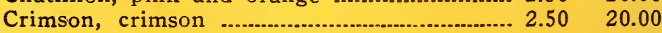

Echo, soft pink ...................................................... $3.00 \quad 25.00$

Edith Cavell, scarlet $\quad 2.50 \quad 20.00$

Ellen Poulsen, bright pink .............................. 2.50 20.00

George Elger, yellow ............................................ $3.00 \quad 25.00$

Golden Salmon, bright orange +.................. $3.00 \quad 25.00$

Greta Kluis, deep pink ................................. $3.00 \quad 25.00$

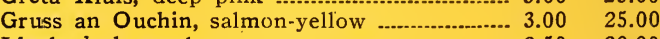

Ideal, dark scarlet

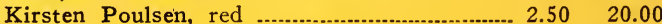

Louise Walter, creamy-pink ................-.-.-..... $3.00 \quad 20.00$

Mrs. Cutbush, silvery rose ......................... $2.50 \quad 20.00$

Orleans, light red $\quad \begin{array}{ll}2.50 & 20.00\end{array}$

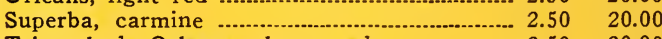

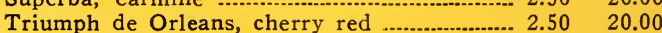

\section{Fifty at the Hundred Rate}

*Prices quoted are for No. 1, 2-year, field-grown, budded plants.

No. $1 \frac{1}{2}$ grade $5 \mathrm{c}$ less

No. 2 grade $10 \mathrm{c}$ less

\section{TREE ROSES}

$\$ 1.00$ Each

Austrian Copper

Betty

Etoile de Hollande

Frau Karl Druschki

General Jack

Golden Emblem

Gruss an Teplitz
Imp. Potentate

Lord Charlemont

Los Angeles

Mme. Butterfly

Mme. C. Testout

Mme. E. Herriot

Miss Lolita Armour
Ophelia

Radiance Pink

Radiance Red

Souv, de Cl. Pernet

Talisman

Wm. F. Dreer 


\section{SPECIAL}

We list the following roses for a grower who specializes in new and rare varieties. Quantity prices on application.

\section{No. $1-75 \mathrm{c}$}

Trigo, intense coppery apricot

\section{No. $1-50 \mathrm{c}$}

Baroness H. Von Geyr, pale flesh and vermillion red Canary, light golden yellow

Conspicuous, scarlet

Conqueror, apricot yellow

Director Rubio, velvety crimson

Dr. Augustin Wibbett, golden yellow

Dr. Heinrich Lumpe, an attractive light rose

Frau. E. Weigand, deep golden yellow

Francy, peach shaded cherry red and gold

Flamingo, geranium red to cerise

Frau. Margarete Oppenheim, intense carmine

Fritz Schrodter, deep crimson

George Dakin, salmon pink

George Howarth, cerise with lemon shading at base

Gladys Benskin, salmon cerise

Helen Fox, Indian yellow

Helen Robinet, yellow and rosy carmine

Impress, flame pink

Joan Cant, salmon

Julio Strahl, capucine red on yellow

La Marechale Petain, brilliant carmine cochineal

Leni Neuss, hydrangea pink

Les Ami de Lille, pale buff

Louis Marie, yellow shaded apricot

Madame A. Lerche, china pink shaded coppery yellow

Madame Charles Allizon, rosy white

Madame Francois Hot, salmon tinted copper pink

Madame Gustave Soupert, dark rose carmine

Marceline, crimson purple

Margaret Anne Baxter, glistening white

Marguerite Chambard, geran. red shading to vermillion Meverouw Dr. L. Crobach, cochineal carmine

Mrs. Hilton Brooks, saffron yellow

Paloma Falco, salmon

Portadown Bedder, orange-yellow.

Sally Tite, brilliant crimson

Souvenir de Pierre Ketten, bright rose

Souvenir D. Pierrot Guillot, yellow and orange

\section{No. $1-40 \mathrm{c}$}

Adelo Crofton, rich apricot

Alexander Marghiloman, creamy white

Amami, fawn pink

Andree Roux, reddish coral

Aphrodite, reddish coral

Cherry, carmine pink and yellow

Diana Cant, carmine red

Dr. Edward Deacon, salmon orange

Dr. Ernest Muhle, soft rose

Dr. Hawksworth, rich crimson

E. Pemberton-Barnes, light pink

Franc Leddy, carmine lake

Francois Allard, salmon pink

Frank Neave, mustard yellow

Frau. Dr. Schricker, carmine

Frau. Fritz Peltzier, orange-carmine

Gaiety, orange-vermillion

Hilda, salmon pink

Hilde Apelt, saffron yellow

Hugo Piller, flesh white

Iris Patricia Green, cherry red

Jeanne Saultier, salmon red

J. C. Thornton, brilliant ruby scarlet

Jules Goujard, brilliant carmine

Konigan Luise, white

Lady Fortevoit, golden yellow
La Mie Au Roy, apricot

Laxton's Standard, cerise

Li Bures, novel red, rose and yellow

Madame Edmond Raynald, creamy yellow

Madame La General Ardouin, carmine yellow

Magnifique, pink

Margaret Spaul, orange

Marie Gouchault, red

Marquise D'Andigne, red

May Whitten, salmon pink

Mme. Henri Queille, shrimp pink

Mrs. Herbert Dowsett, flame pink

Mrs. S. Paton, orange

Nuntius Pacelli, white

Orange Perfection, orange red

Portadown, deep crimson

Robin Hood, musk and cherry red

Ronald Tooke, nearly "black"

Rose Berkeley, rosy salmon

Rupert Brooke, fawn pink

Sarah Wright, pink

Souvenir de Charles Gouveneur, salmon

Souvenir de Gustave Shickels, rosy scarlet

Souvenir de Nungesser, red

\section{No. $1-30 \mathrm{c}$}

Alicd Stern, white

Antonio Relleri De Peluffo, brilliant red

Comtesse $\mathrm{De}$ Castilleja, orange-vermillion

Everest, ivory white

Fascination, rosy cerise

Gela Gnau, apricot

Golden Gleam, buttercup yellow

Gouvlinder, orange

Hilda Steinert, reddish salmon

Ivan Davis, sport "Butterfly"-darker

Kard Nal Schultze, crimson

Lady Love, fawn pink

Madelle Bep Van Rossem, pure yellow

Maud Gladstone, bright pink

Miss Theresa Morley, carmine cerise

Mrs. G. A. Wheatcroft, coppery pink

Oliver Mee, deep salmon

Souvenir D'Alexandre Bernaix, crimson

Souvenir De Angele Op De Beeck, yellow

Souvenir de Damade Pidoux, yellow

The Princess Elizabeth, sport "Queen Alexandra"

Villa Pia, dark crimson

Waltham Cross, crimson scarlet

\section{No. $1-25 \mathrm{c}$}

Arthur Cook, deep crimson

Belle Cuivree, coral with yellow

Cecil, golden yellow

Christinc Prior, golden yellow

Eric Holroyd, vermillion with gold

Golden Salmon, orange

Gooiland Glory, cherry red

Julia Countess of Dartrey, rose and yellow

Lady Helen Maglona, crimson

Lady Mary Elizabeth, carmine

Lucie Fernand David, white

Niadame Chamonton Murgue, orange-vermillion

Madame Leon Guinotte, old gold

Marietta Silva Tarouca, climber, rose

Mevrouw L. C. Van Gendt, salmon apricot

Philomene Pollaert, crinson-orange

President F. A. Des Tombs, golden

Princess Elizabeth of Greece, orange

Princess Marie Jose, orange-vermillion

Royal Scot, yellow with crimson

Rufus, pure crimson

Salmon Spray, salmon pink

Star of Prague, deep red

Surprise, salmon pink

Victor Waddilove, salmon orange

W. A. Bilney, yellow cerise 


\section{Hardy Perennial Phlox}

Every garden to be complete should have a planting of Phlox.

Albion-Large pure white flowers with faint purplish-red eye ....................................................\$6.00

Athis, tall-Light salmon; tall ................................ 7.00

Athis, dwarf-Same flower as above, only shorter growing

Bryn Mawr-Orchid pink, with faint red eye and light halo

Champs Elyssee-Rich dark crimson ....................... 9.00 9.00

Colridge-Light rosy crimson with clear claret eye 7.00

Dinty Moore-Clear light red 9.00

Eclaireur-Carmine violet-red with lighter center;

drought resistant

8.00

Elite-Light lavender, delicately mottled ................. 9.00

Etoile de Feu-Red center shading to white outer petal 9.00

Fraulein von Lassburg-An old favorite among the whites

Ideal-Purple red with crimson eye ......................... 7.00

Julia Bonnell-Shell pink with red eye ..................... 6.00

La Petite-White with red eye; large flower clus

ters

La Reine-Bluish white

La Rouge-Deep rose red

La Vague-Pure mauve with reddish eye

Miss Lingard-Fine, free flowering white; earliest

of all ............................................................................. bushy

6.00

Pantheon-Deep rose, tall and showy; fine for

mass effects .............................................................. 6.00

Peachblow-Shell pink, rose center .......................... 7.00

Pearl-(Syn. Jeanne d'Arc). Creamy white; tall........ 6.00

Queen-Large compact head of pure white flowers;

latest of whites .................................................. 6.00

Rhynstrom-Rose pink ............................................ 6.00

Rosenburg-Clear pink, heavy bloomer; dwarf

bushy foliage ............................... 6.00

Selma-Lilac rose, with crimson eye ...................... 9.00

Shelton-Dark pink, red eye with light halo............ 9.00

Special French-Large flowered, pink with violet

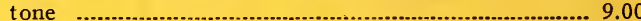

Thor-Large flowers. of deep salmon pink, crimson

eye ................... 7.00

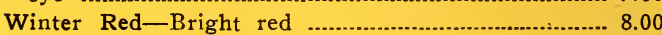

Our prices are extremely low for quality stock and are based on cash with order or C. O. D. unless in. cluded with order for other stock. When ordering, state whether we shall ship by freight, express or parcel post.

\section{Lining Out Stock, Perennials, Bulbs, Etc.}

We shall be pleased to quote specially on a general list of lining out stock in deciduous shrubs, conifers, broad-leaved evergreens, vines, climbers, perennials,' bulbs, etc., not listed herein.

\title{
THIS CATALOG INCLUDES
}

\author{
Fruit Trees \\ Berry Fruits \\ Shade Trees \\ Shrubs Flowering \\ Conifers \\ Hedge Plants
}

Nut Trees

Seedling Stocks

Flowering Trees

Shrubs Evergreen

Vines Flowering .

Roses and Perennials

\author{
Ask'us for list of \\ Lining Out Stocks \\ Russellville Nurseries \\ East 96th and Burnside, Streets, \\ Portland, Oregon
}



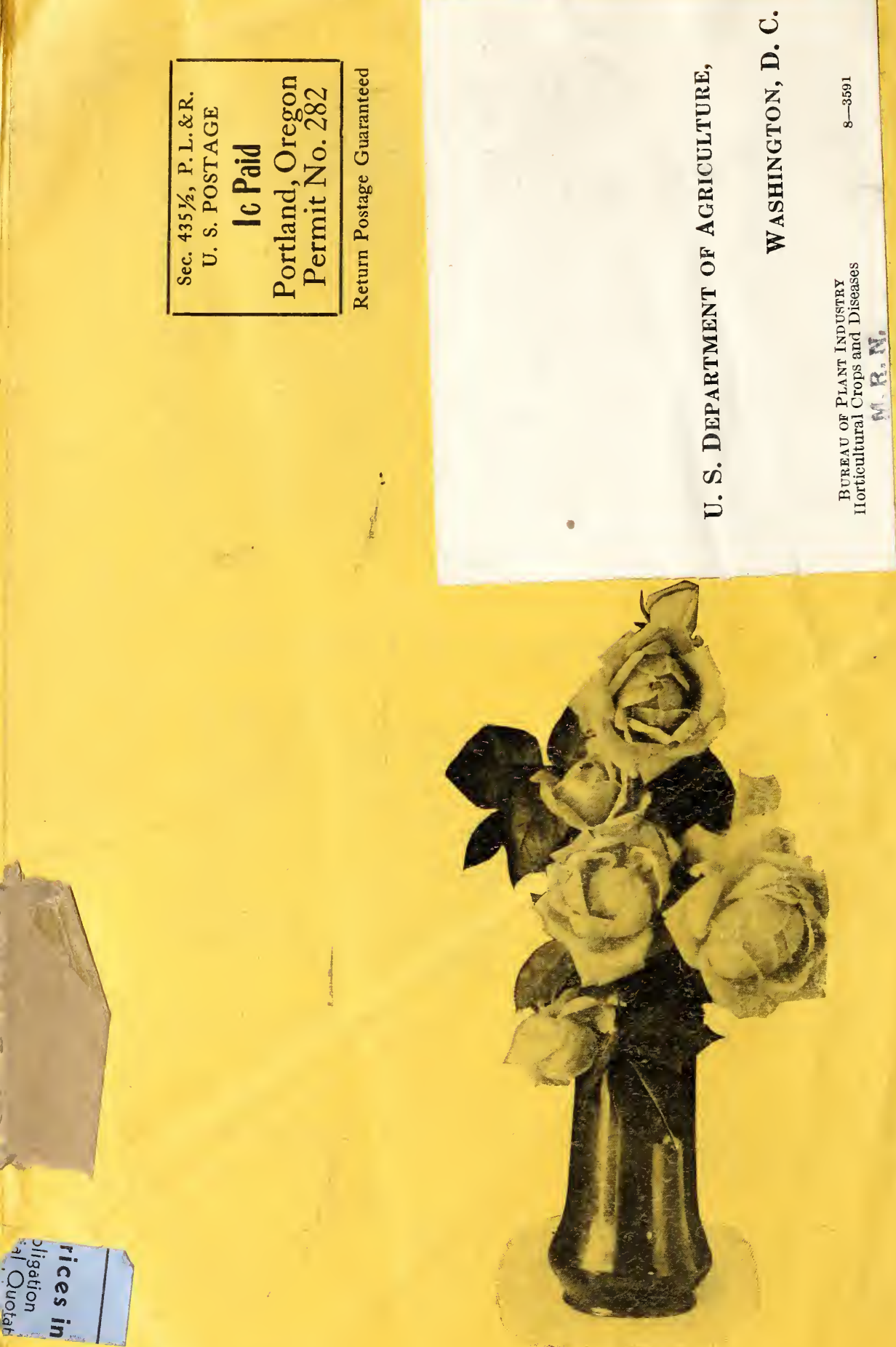

We shall always endeavor to render that dependable type of service which one comes to expect from a 40 year old institution.

\section{Russellville Nurseries}

East 96th and Burnside Streets, Portland, Oregon 